\title{
Korean Legal EducAtion for the Age of Professionalism: SUGGESTIONS FOR MORE CONCERTED CURRICULA
}

\section{YOUNG-CHEOL K. JEONG*}

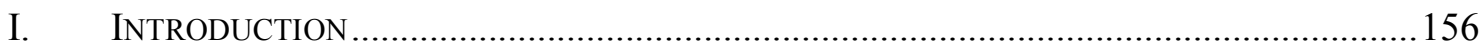

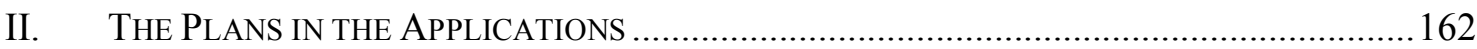

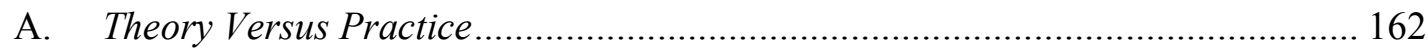

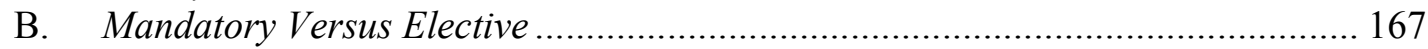

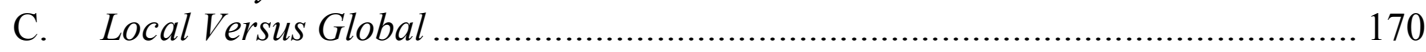

III. REASONS FOR More Practical, Global, ElECtive AND NON-Litigation COURSES ...

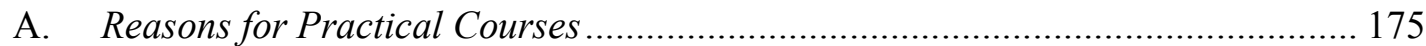

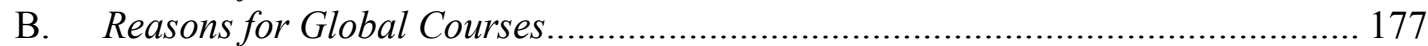

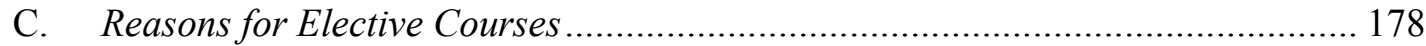

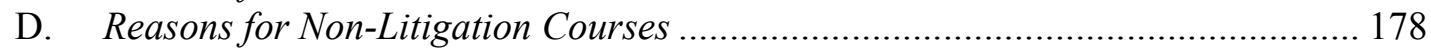

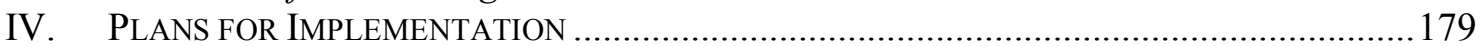

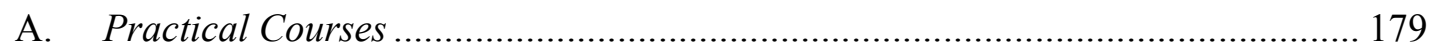

i. $\quad$ Number and Content of Classroom Practical Courses.................................. 179

ii. Practical Courses Outside the Classroom ..................................................... 182

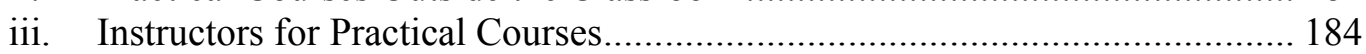

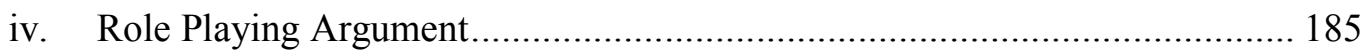

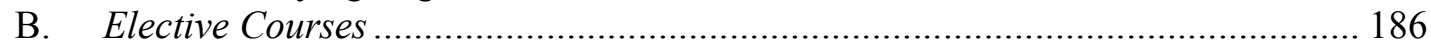

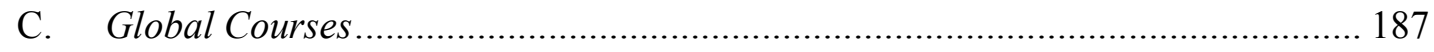

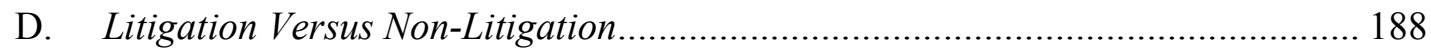

V. StRATEgIES FOR SUCCESSFUl INSTITUTIONALIZATION OF LAW SCHOOLS IN KOREA .. 189

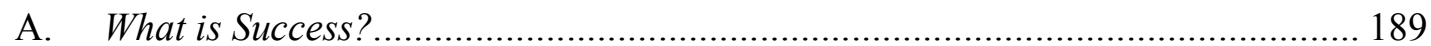

B. Change in Values: The Lawyer as Professional ................................................ 189

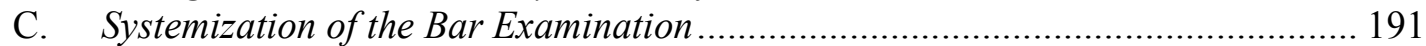

D. Programming of Legal Education ..................................................................... 192

E. Addressing Concerns About the Quality of Lawyers: Continuing Legal Education as Part of Law School Programs .............................................................................. 192

F. Adaptation of General Graduate Study Programs within Law Schools................ 193

G. Alliance with Foreign Law Schools ........................................................... 194

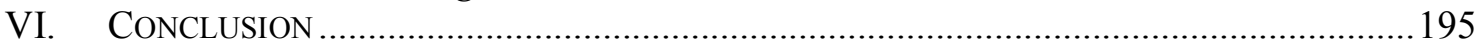

\footnotetext{
* Professor, Yonsei Law School. LL. B., Seoul National University, 1978; LL.M., Seoul National University, 1982; LL.M., Columbia Law School, 1984; J.D., Columbia Law School, 1986. Member of the Korea, Illinois and Washington, D.C. bars. Prof. Jeongoh Kim of Yonsei Law School made extremely careful comments. I am also grateful for comments from Prof. Alexander Loke of National University of Singapore. Special thanks to two Yonsei Law School students for their help: Seung-Gwan Kang and Joo-Mi Kim.
} 


\section{INTRODUCTION}

Korea has ushered in a new era of legal education through a revolutionary change in its goals, institutions, and methodology. The goals of legal education are not to let as many students as possible pass the ever-competitive national bar examination by memorizing the substance of basic laws, but rather to ensure that students can think like lawyers. Rather than knowing in detail the substance of many particular laws, such students will possess more basic knowledge of the core of the Korean legal system. The main institutions for legal education in Korea are no longer the four-year colleges and the Judicial Research and Training Institute (“JRTI”), but chartered three-year graduate law schools. The methodology of legal education is not to subject students to one-sided lectures and urge them to memorize dogma, but to question the students about the facts and issues relating to specific situations and let them create legal solutions.

After long debates about legal education reform dating to the early 1990s, the Korean National Assembly passed the Law for the Establishment and Operation of Law Schools (the "Law School Law") near midnight on July 3, 2007. ' In two short years, the Law School Law would see a number of events and revisions. ${ }^{2}$ The Law School Law was promulgated by then President Roh on July 27. Article 1 of the Addendum provided that the Law School Law would take effect two months after promulgation, on September 28, 2007. ${ }^{3}$ Article 2 of the Addendum also set the target date for the admission of new students as March 1, 2009. On

\footnotetext{
${ }^{1}$ Beophak jeonmun taehakweon seolch'i unyeong e gwanhan beopryul [Law for Establishment and Operation of Law Schools], Law No. 8544 of 2007 [hereinafter Law School Law]. For the sketchy history of legal education in Korea before the passage of the Law School Law, see Jae Won Kim, The Ideal and the Reality of the Korean Legal Profession, 2 Asian-PAC. L. \& POL'Y J. 34 (2001); Kyong-Whan Ahn, Law Reform in Korea and the Agenda of “Graduate Law School,” 24 WiS. INT’L L. J. 223 (2006); Sang-Hyun Song, Legal Education in Korea and the Asian Region, 51 J. LEGAL EDUC. 398, 398-401 (2001); Soogeun Oh, Globalization in Legal Education of Korea, 55 J. LEGAL EDUC. 525, 525-526 (2005); and Tom Ginsburg, Transforming Legal Education in Japan and Korea, 22 PENN. ST. INT'L L. REV. 433, 438-439 (2004).

${ }^{2}$ For the process in Japan, see Sabrina Shizue KcKenna, Proposal for Judicial Reform in Japan: An Overview, 2 ASIAN-PAC. L. \& POL'Y J. 122 (2001).

${ }^{3}$ Law School Law, Arts. 27 to 37, provisions regarding evaluation, took effect on Jan. 1, 2009.
} 
September 28, 2007, the Enforcement Decree under the Law School Law (the "Law School

Law Enforcement Decree") was promulgated. On October 30, the Ministry of Education and

Human Resources (the "Education Ministry") released detailed evaluation guidelines (the “Guidelines") for law school applications. ${ }^{4}$ By November 30, 41 law colleges had filed applications for law school charters. On January 30, 2008, the Legal Education Committee preliminarily allocated 2,000 students to 25 law colleges. ${ }^{5}$ In March of that year, the Law School Association (the "Association") formulated the basic tenets of admission standards and major timelines. On August 24, the Association administered the first Legal Education Eligibility Test (“LEET”). On August 30, government charters were granted to twenty-five colleges. $^{6}$ In October, approximately 10,000 applications were filed. ${ }^{7}$ In November, law schools had one or two interview sessions with applicants. In early December, two-thousand students were officially selected. On March 1, 2009, these students started their legal studies

\footnotetext{
${ }^{4}$ Official Letter from the Korean Education Ministry to the twenty-five law schools (Sep. 2, 2008).

${ }^{5}$ Sixteen colleges failed to secure the law school charter and thus continue to maintain their undergraduate law colleges. The twenty-five successful law schools are: Kangwon National University (“KNU”), KonKuk University (“KKU”), KyungPook National University (“KPNU”), Kyung-Hee University (“KHU”), Korea University ("KU”), Dong-A University ("DAU”), Pusan National University ("PNU”), Sogang University ("SU"), Seoul National University ("SNU”), University Of Seoul ("UOS”), Sung-Kyun-Kwan University ("SKKU”), Ajou University (“AU”), Yonsei University ("YLS”), YeungNam University ("YNU”), WonGwang University ("WU"), Ewha Woman's University ("EWU”), Inha University ("IU"), ChonNam National University ("CNNU”), Chonbuk National University ("CBNU”), Jeju National University ("JNU”), Chung-Ang University ("CAU”), Chungnam National University( "CNU”), ChungBuk National University ("CBU”), Hankook University of Foreign Studies("HUFS"), and Hanyang University ("HU”). In the cases of ChonNam National University ("CNNU"), ChonBuk National University ("CBNU”), Chungnam National University ("CNU") and ChungBuk National University("CBU”), their abbreviations in this article are modified from the official ones to avoid confusion among them. For the number of students for each law school, see infra Table 1.

${ }^{6}$ The charter will be reviewed after three years, probably early in 2012 , while the new graduate law schools are not allowed to recruit new students for their undergraduate law colleges. This is the biggest difference of law schools in Korea from those in Japan. For Japanese reform, see Kahei Rokumoto, Legal Education, in LAW IN JAPAN 190, 215-227 (Daniel H. Foote et al. eds., 2007). Japanese reform seems to have been a complete fiasco as many local law schools wish to close their doors. See Luke Nottage, Reformist Conservatism and Failures of Imagination in Japanese Legal Education, 2 ASIAN-PAC. L. \& POL'Y J. 28 (2001). As for other attempts to combine civil law tradition with common law needs, see Yves-Marie Morissette, McGill's Integrated Civil and Common Law Program, 52 J. LegAl EduC. 12 (2002), and Jutta Brunnee, The Reform of Legal Education in Germany: The Never-Ending Story and European Integration, 42 J. LEGAL EDUC. 399 (1992).

${ }^{7}$ As in 2008, an applicant can apply for up to two schools in 2009. See KorEAN AsSOCIATION OF LAW SCHOOLS, PUBLIC NOTICE OF LEET AND ADMISSION STANDARDS FOR 2009, available at http://info.leet.or.kr/.
} 
at twenty-five law schools with high hopes. ${ }^{8}$ On April 29, 2009, the Bar Examination Law passed the Korean National Assembly, which took effect three months after promulgation by the President, on August 29, 2009. ${ }^{9}$ Along with the newly created law school plus perfunctory bar examination system whose first graduates will gain admission to the Bar in $2012,{ }^{10}$ the traditional national bar exam will continue till $2017,{ }^{11}$ and thus JRTI will continue to operate its current practical training programs till $2020 .^{12}$ Although the debate over law school reform has taken the past two decades, the speed of implementation has been dazzling.

The driving force behind such sweeping changes, among other things, was competitive tensions among universities; a law school charter is an invaluable and prestigious franchise granted by the government, and thus every law college was expected to marshal all available financial, political, and emotional support from the university administration and local residents. Many local law colleges which had no or few bar exam passers in the past would be able to produce licensed lawyers from their law schools at last. While this upcoming change indicates the past privileges of licensed lawyers, it also signals the remoteness of legal services from the general public. Thus, the Korean bar's acceptance of the proposition that more licensed lawyers should be produced was urged by the public,

\footnotetext{
${ }^{8}$ The association's website has accumulated general information on the history of law school system. See id.

${ }^{9}$ Byeonhosa Siheumbeop [Bar Examination Law]. Law No. 4675 of 2009.

${ }^{10}$ Although the Bar Examination Law itself does not stipulate the minimum passage rate, $80 \%$ is an unofficial, but widely accepted passage rate. If so, sixteen-hundred new lawyers from law schools will be produced in the spring of 2012, along with one-thousand graduates of the traditional Judicial Research and Training Institute.

${ }^{11}$ The number successful takers of the traditional bar exam in 2009 was one-thousand. As they will become licensed after a two-year education program at JRTI, they will come into the market in 2012 at the same time when 2,000 law school students graduate the twenty-five new graduate law schools. If the passage rate is $70 \%$, the number of new lawyers in 2012 will be about twenty-four hundred, the biggest number in Korean judicial history. In 2010, eight hundred newly admitted lawyers will come from the traditional bar exam. In 2011, seven hundred will be selected. See Press Release, Korean Ministry of Justice (Apr. 17, 2008), available at http://www.moj.go.kr/HP/COM/bbs_03/BoardList.do. More recently, for 2012, five hundred candidates, and for 2013, three hundred candidates will be selected. For 2014, 2015, 2016 and 2017, the number of candidates to be selected is an open issue. I am not sure what the future holds for those undergraduate law colleges who failed to receive a graduate law school charter. For the situation in Japan, see Gerald Paul McAlinn, Reforming the System of Legal Education: A Call for Bold Leadership and Self-Governance, 2 ASIAN-PAC. L. \& POL'Y J. 15 (2001).

${ }^{12}$ Bar Examination Law, Addendum Art. 4.
} 
politicians, and law professors, most of whom did not pass the bar examination. ${ }^{13}$ This outside pressure might be natural, as the legal profession, despite having privileges such as a monopoly on the ability to practice law, failed to provide important social services, such as regulating social transactions and securing justice. ${ }^{14}$

Considering the extremely short time period within which law colleges have had to prepare their application documents, the planned applications will have to be changed to accommodate certain practical problems. Because law schools are subject to review in 2012, however, they will have to conform to their initial plans for higher evaluation scores to the review process. ${ }^{15}$ Nevertheless, most law schools prepared their plans under the assumption that the maximum number of students per school, one hundred fifty, would be realized, which could be used as a pretext for deviation from the plan. This article will address possible desirable changes to curricula in 2010 and 2011. Discussions in this paper, however, assume that law school curricula will be separated completely from preparation for bar examinations. Furthermore, each law school is assumed to be financially viable for the next two years, and thus the impact of financial considerations on the implementation of the most ideal curricula are not accounted for. It is also assumed that any deviation from the plan will have no adverse effect on the evaluation of law schools in 2012.

As the subject of curricula as a whole is an issue which could determine the future of the Korean legal education system, this paper focuses on the following dichotomies: theory

\footnotetext{
${ }^{13}$ The political will of the ruling party, supported by the public and certain political judges and prosecutors who agreed to such moves, was of course the initiating force.

${ }^{14}$ William M. Sullivan et al., Educating LaWyers: Preparation for the Profession of LaW 21 (2007) [hereinafter CARNEGIE REPORT]. For one explanation of the reasons for Japanese legal education reform, see Robert F. Grondine, An International Perspective on Japan's New Legal Education System, 2 ASIAN-PAC. L. \& POL'Y J. 1, 6 (2001) (arguing that Japan reformed its legal education to address a shortage of lawyers). I find lacking the persuasiveness of the "too-few lawyers" hypothesis.

${ }^{15}$ Law School Law, Arts. 27 et seq.; Law School Law Enforcement Decree, Arts. 18 et seq. The first re-evaluation shall be performed during the fourth year after the first year when the new students are admitted, which is 2012. Thereafter, every five year evaluation is expected. Each law school is also required to submit its internal audit report to the Evaluation Committee two years prior to the expected evaluation. Thus, in 2010, law schools are required to execute internal audits and submit their outcome to the Evaluation Committee.
} 
versus practice; mandatory versus optional; local versus global; and dispute resolution versus dispute prevention. ${ }^{16}$ This framework of dichotomies presents itself as the indicia of lawyers as professionals. "A professional lawyer is an expert in law pursuing a learned art in service to clients and in the spirit of public service and engaging in these pursuits as part of a common calling to promote justice and public good."17 Practical, elective, global, and dispute prevention education better foster the legal expertise necessary for helping clients as well as a firm commitment to the welfare of the larger community than does dogmatic, mandatory, local, and gun-for-hire education. ${ }^{18}$ On the basis of these assumptions and foci, Part II of this paper reviews the status of the curricula in the application documents and compares them with the current practical education administered by JRTI. Part III provides an overview of the goals and reasons for practical, global, and elective education in law schools. Part IV is an evaluation of Part II in light of the goals and reasons presented in Part III, where I examine what is missing from current curricula and is thus desirable for future curricula. This paper proposes a more desirable way of executing the plans for the upcoming years until the first graduates of the 25 newly-chartered law schools turn up in February 2012. Furthermore, it aims to prevent any distortion in the criteria for the successful evaluation of law schools in $2012^{19}$ and thus further the expansion of a flawed system in the future. ${ }^{20}$

${ }^{16}$ For the changes in Singapore since 2001, see Alexander Loke, Forging a New Equilibrium in Singapore Legal Education, 24 WIS. InT'L L. J. 261 (2006). For the changes in Japan since 2004, see Katsumi Yoshida, Legal Education Reforms in Japan: Background, Rationale, and Goals To be Achieved, 24 WIS. INT'L L. J. 209 (2006); see also Rokumoto, supra note 6. Even in the United States, discussions for reform are incessant. See, e.g., Jonathan D. Glater, Training Law Students for Real-Life Careers, N.Y. TIMES, Oct. 31, 2007; Alan Watson, Legal Education Reform: Modest Suggestions, 51 J. LegAL EduC. 91 (2001).

${ }^{17}$ AMERICAN BAR ASSOCIATION, REPORT OF THE PROFESSIONALISM COMM. OF THE AMERICAN BAR ASSOCIATION's SECTION OF LEGAL EDUCATION AND ADMISSIONS TO THE BAR 6 (1996).

${ }^{18}$ For a discussion on professional identity and purpose, see CARNEGIE REPORT, supra note 14, at $126-29$.

${ }^{19}$ On Dec. 5, 2009, the Education Ministry held a public hearing about draft evaluation plan at the Lawyers Building. The plan would be finalized by the Law School Evaluation Committee at the Korean Bar Association. The plan lists eight factors: goal of education; entrance exam administration, curriculum, faculty, student body, education facilities, education and research supports, and degree programs. See Korean Bar Assoc., Beophak jeonmun taehakweon p'yeonggagi chunan jakseongeul uihan kongch'eonghoi [Notice of Hearings for the Creation of Standards for Law School Assessment] (Nov. 30, 2009), available at http://www.koreanbar.or.kr/notice/index_read.asp?idx=796\&Page=1\&strSearchList=\&strSearchWord=. 
Ultimately, the curricula for 2010 and 2011 will determine the future of the students and their instructors' record in history.

I aver that, despite the good faith efforts on the part of every law school, the curricula at these schools are disconcerting. They are just window-dressing. They have neither strategies nor plans of action. For immediate and long-term success, more practical courses should be added to the third-year curriculum. An environment where diverse practical teaching such as clinics and moot competitions can flourish should be fostered. The mandatory courses should be limited to the core of the system in the first-year courses. For global courses, so long as Korean law schools do not teach Chinese, Japanese, American, Russian, Southeast Asian, Islamic, Jewish, Latin American, and other foreign laws with full time faculty members on their campus, more students should be encouraged to go abroad for one semester. Although dispute resolution is the traditional role of lawyers, a more atypical way of preventing and resolving disputes should be part of the educational discourse.

Finally, Part V addresses specific strategies for implementing these changes in 2010 and 2011. When the critical 2012 review of the current system reaches law schools, the legal profession in Korea should be ready to expound their visions for the next phase and plans of action to the public, whom the legal profession is supposed to serve. The eye of the storm, the number of students for each law school, should be revisited in 2012 and increased to at least three thousand. It is true, however, that such a move would have to pass many rocks and countercurrents, such as broken promises in the applications, financial difficulties,

See also Yong Tae Kwon, Roseuk'ul p'yeongga, 50\% isang hapgyeok, haksaeng 30\% changhak hyet'aek gijun jeshi [Law School Evaluation: 50\% bar exam passage rate \& 30\% scholarship proposed], BEOPRYUL SHINMUN (Dec. 12, 2009), available at http://www.lawtimes.co.kr/LawNews/News/NewsContents.aspx?kind=\&serial=50280.

${ }^{20} \mathrm{~A}$ big question that is not discussed in this paper is how best to teach law. For the discussions on Japan, see Mark Levin, The American Kaizen of Law Teaching, 2 AsIAN-PAC, L. \& POL'Y J. 6 (2001). See also Carl E. Schneider, On American Legal Education, 2 ASIAN-PAC. L. \& POL'Y J. 76 (2001). 
student expectations, legislative constraints, schisms among faculty members, objections and intrusions from the vested-interest holders, and a general lack of preparation.

\section{The Plans IN The ApPlications}

\section{A. Theory Versus Practice}

Traditional legal education in Korea has been divided into two parts: (i) theory and/or bar examination preparation ${ }^{21}$ at law colleges; and (ii) practical education and training at the JRTI. The practical education at the JRTI is targeted at students whose goal is to enter the courts and public prosecutor's offices as bureaucrats. Table 3 breaks down the courses offered by the JTRI for four semesters of classroom lectures and on-the-job training by judges and public prosecutors. Practice courses for drafting court decisions and indictment letters account for forty-four credits out of the fifty-seven credits received during JRTI training. All the faculty members are temporarily posted at the JRTI from courts and public prosecutor's office. So-called theoretical courses are, in actuality, all internalization of court decisions. Most participants' aim for appointments as judges or public prosecutors, like their teachers. ${ }^{22}$ Now, because practical education will have to be merged into the curriculum of graduate law schools, the need to expand practical courses or seminars will increase. Thus, the Law School Law should require that one fifth of the faculty be licensed lawyers who have five or more years of practical experience. ${ }^{23}$ The Evaluation Guidelines, proclaimed by the Education Ministry, also awarded ten points for diversity of courses combining legal theories with legal practice if five or more such courses are offered. ${ }^{24}$ Thus many law schools

\footnotetext{
${ }^{21}$ Most law colleges have been allocating enough resources to the students who prepare the bar exam. At YLS, immediately next to the faculty offices, students preparing the bar exam occupy special study space and resting lounges.

${ }^{22}$ YounG-SOO KANG, JRTI AND LAW SCHOOL EDUCATION (2008) (on file with author).

${ }^{23}$ Law School Law, Art. 16, Para. 4.

${ }^{24}$ Guidelines, Item 3.6.2. They illustrate civil/corporate/criminal case studies, corporate litigation law and practice, international investment law and practice and medical law and practice. The diversity of courses is also assessed in terms of interdisciplinariness and the combination of substantive and procedural laws.
} 
proposed practice courses in their application documents. The number of credits for theory courses vis-à-vis practice courses for each law school is given in Table $1 .^{25}$ Table 2 lists all of the practical courses to be offered by law schools.

The ratio of theory courses to practice courses ranges from four to twenty-six. The number of practice courses is limited to the range of ten to seventy-six. Most of practice courses are a repetition of the case studies commonly undertaken during senior years at undergraduate law colleges ${ }^{26}$ which should be incorporated into the substantive law courses for second-year graduate law students. Furthermore, many law schools tend to regard the term "law practice" as litigation, because many practice courses cover civil, criminal, constitutional, and tax litigation. Some exceptional law schools include contract drafting, transactional negotiations, tax planning and clinic courses to the category of practice courses.

Table 1: Number of Credits for Theory Courses v. Practice Courses ${ }^{27}$

\begin{tabular}{|c|c|c|c|c|c|c|}
\hline $\begin{array}{l}\text { Name of } \\
\text { School }\end{array}$ & $\begin{array}{l}\text { Total } \\
\text { Number of } \\
\text { Courses }\end{array}$ & $\begin{array}{l}\text { Theory } \\
\text { Course Credit }\end{array}$ & $\begin{array}{l}\text { Practice } \\
\text { Course } \\
\text { Credit }\end{array}$ & Ratio & $\begin{array}{l}\text { Number } \\
\text { of } \\
\text { Students }\end{array}$ & $\begin{array}{l}\text { Number } \\
\text { of } \\
\text { Faculty }\end{array}$ \\
\hline $\mathrm{KNU}^{29}$ & 123 & 216 & 47 & 4.6 & 40 & 30 \\
\hline KKU & ND & $\mathrm{ND}^{30}$ & ND & ND & 40 & 28 \\
\hline KPNU & 143 & 334 & 50 & 6.7 & 120 & 36 \\
\hline KHU & 148 & ND & ND & ND & 60 & 31 \\
\hline KU & ND & ND & ND & ND & 120 & 51 \\
\hline DAU & 113 & 270 & 36 & 7.5 & 80 & 41 \\
\hline PNU & 122 & 321 & 22 & 14.6 & 120 & 37 \\
\hline SU & 115 & 278 & 41 & 6.8 & 40 & 41 \\
\hline SNU & 190 & ND & ND & ND & 150 & 57 \\
\hline UOS & 138 & 262 & 10 & 26.2 & 50 & 27 \\
\hline SKKU & ND & ND & ND & ND & 120 & 42 \\
\hline
\end{tabular}

${ }^{25}$ All the data in this paper is from the Association and websites of chartered law schools.

${ }^{26}$ These courses apparently originated from Übungs (translated as drills, exercises or practice) in German law colleges.

${ }^{27}$ Some law schools do not categorize practice courses. Regardless of whether they are categorically distinguished or not, as long as the course name includes practice, I count them as practice courses.

${ }^{28}$ The number of the faculty is based on the websites of applicable law schools; however, this figure may not always be accurate as certain law schools try to exaggerate the size of their faculties by including adjunct practitioners. For foreign faculty numbers, see infra note 40 and accompanying text.

${ }^{29}$ For the full names of the listed law schools, see supra note 5.

${ }^{30}$ Some law schools do not disclose their curriculum on their websites. 


\begin{tabular}{|l|l|l|l|l|l|l|}
\hline AU & 154 & 257 & 49 & 5.2 & 50 & 41 \\
\hline YLS & 153 & 377 & 40 & 9.4 & 120 & 50 \\
\hline YNU & 106 & 282 & 14 & 20.1 & 70 & 29 \\
\hline WU & 135 & 268 & 12 & 22.3 & 60 & 28 \\
\hline EWU & 196 & 505 & 38 & 13.3 & 100 & 48 \\
\hline IU & 133 & 290 & 77 & 3.8 & 50 & 59 \\
\hline CNNU & 122 & 250 & 41 & 6.1 & 120 & 61 \\
\hline CBNU & 128 & 278 & 69 & 4.0 & 80 & 30 \\
\hline JNU & 107 & 297 & 32 & 9.3 & 40 & 24 \\
\hline CAU & 150 & 376 & 47 & 8 & 50 & 47 \\
\hline CNU & 99 & 195 & 50 & 3.9 & 100 & 38 \\
\hline CBU & 97 & 252 & 33 & 7.6 & 70 & 33 \\
\hline HUFS & 160 & ND & ND & ND & 50 & 30 \\
\hline HU & 156 & 432 & 20 & 21.6 & 100 & 47 \\
\hline
\end{tabular}

ND: Not Disclosed.

Table 2: Content of Elective Practice Courses ${ }^{31}$

\begin{tabular}{|l|l|}
\hline KNU & $\begin{array}{l}\text { Theory \& Practice of Criminal Procedural Law; Theory and Practice of Labor Law; } \\
\text { Theory and Practice of Environmental Litigation;Theory and Practice of } \\
\text { Constitutional Law; Theory and Practice of Civil Law I, Theory and Practice of } \\
\text { Administrative Law; Theory and Practice of Civil Law II, Theory \& Practice of Mergers } \\
\text { \& Acquisitions; Theory and Practice of Criminal Law; Practice of Competition } \\
\text { Law; Anglo-American Law Practice; Practice of Insurance \& Maritime Law; } \\
\text { Practice of Social Law; Commercial Law and Practice }\end{array}$ \\
\hline KKU & ND \\
\hline KPNU & $\begin{array}{l}\text { Constitutional Litigation Practice; Administrative Litigation Practice; Family Law } \\
\text { Litigation Practice; Criminal Proceedings Practice; Property Crime Practice; M\&A } \\
\text { and Corporate Finance Practice; International Business Transactions Practice; } \\
\text { Human Rights Practice; Labor Dispute Practice; IT International Transaction } \\
\text { Practice; International Transaction and WTO/TRIPs Practice; Debt Collection } \\
\text { Practice; Civil Contract Practice; Technology Transfer and Licensing Practice }\end{array}$ \\
\hline KHU & $\begin{array}{l}\text { Civil Matters Private Practice; Public Law Private Practice; Administrative Law } \\
\text { Practice; Criminal Matters Private Practice; Diverse Civil Matters Practice; Diverse } \\
\text { Civil/Commercial Matters Practice; Commercial Arbitration Practice; Diverse } \\
\text { Public Law Practice; Diverse Criminal Practice; Family Disputes Practice; } \\
\text { International Contracts Drafting Practice }\end{array}$ \\
\hline KU & ND \\
\hline DAU & $\begin{array}{l}\text { Lawyering, Leadership \& Communication Skills for Lawyers; Legal Clinic; } \\
\text { Administrative Litigation Practice Seminar; International Commercial Arbitration } \\
\text { Practice; Real Estate Registration and Auction Practice; Chinese Conflict Law } \\
\text { Practice; Public Law Practice; Civil Matters Practice; Crim. Law Practice; E-Trade } \\
\text { and Litigation Practice }\end{array}$ \\
\hline
\end{tabular}

${ }^{31}$ Even if the name of the courses in English sounds rather awkward, the original title given by each law school is retained. Translation of course names conforms to the literal meaning of the title. 


\begin{tabular}{|c|c|}
\hline PNU & $\begin{array}{l}\text { Investigation Procedure Practice; Civil Matters Litigation Practice; Criminal Hearing } \\
\text { Practice; Constitutional Litigation Practice; Administrative Litigation Practice; IP } \\
\text { Disputes and Practice; Bankruptcy Law and Practice }\end{array}$ \\
\hline SU & $\begin{array}{l}\text { Theory and Practice of Fair Trade Law; Civil Proceedings Practice (Property Law); } \\
\text { Public Law Practice; Civil Proceedings Practice (Obligation/Tort Law); Corporate } \\
\text { Matters Practice; Consumer Law Theory and Practice; International Litigation } \\
\text { Practice; Criminal Proceedings Practice; Civil Proceedings Practice (Family } \\
\text { Disputes); M\&A Practice; Environment Disputes and Litigation Practice; Theory and } \\
\text { Practice of Mediation of Criminal Matters }\end{array}$ \\
\hline SNU & $\begin{array}{l}\text { Law and Practice of Finance Regulations; Corporate Finance Law and Practice; } \\
\text { Administrative Proceedings Practice; Constitutional Litigation Practice; } \\
\text { International Investment Practice; Prosecution Practice }\end{array}$ \\
\hline UOS & Life Path as Lawyers; Civil Practice; Criminal Practice \\
\hline SKKU & $\begin{array}{l}\text { Public Law Litigation Practice; Criminal Proceedings Practice; Civil Proceedings } \\
\text { Practice; Corporate Litigation Practice; Jury Charge Practice; IP Prosecution and } \\
\text { Litigation Practice }\end{array}$ \\
\hline $\mathrm{AU}$ & $\begin{array}{l}\text { Legislation Practice; Civil Matters Private Practice; Preservation Proceeding } \\
\text { Practice; Criminal Defense Practice; Corporate Advising Practice; Tax Litigation } \\
\text { Practice; Public Litigation Practice; Small Medium Enterprises Legal Practice } \\
\text { Internship; Prosecution Practice; Criminal Evidence Law and Investigation } \\
\text { Practice; Corporate Litigation Practice; International Trade Litigation Practice; } \\
\text { Small Medium Enterprises Tax Management Practice; Small Medium Enterprise } \\
\text { Labor Consulting Practice; Civil Proceedings Practice; White Crime Theory and } \\
\text { Litigation Practice; Criminal Proceedings Practice; Industrial Accident Claim } \\
\text { Practice; International Trade and Tax Practice; Family Dispute Litigation Practice; } \\
\text { Foreign Laborer Human Rights Claim Practice }\end{array}$ \\
\hline YLS & $\begin{array}{l}\text { Public Law Application; Civil Law Application; Criminal Law Application; } \\
\text { Commercial Law Application; Public Law Disputes Practice; Civil Proceedings } \\
\text { Practice; Criminal Proceedings Practice; International Contract Practice; Law and } \\
\text { Practice on Trans-border Flow of Goods; Technology and Capital; Finance and Tax } \\
\text { Practice; IP Licensing Practice }\end{array}$ \\
\hline YNU & Civil Law Practice; Administrative Litigation Practice; Criminal Law Practice \\
\hline WU & Witness Questioning Technique; Oral Argument; Fact Finding; Negotiations \\
\hline EWU & $\begin{array}{l}\text { Administrative Litigation Practice; Medical Civil Disputes Practice; } \\
\text { Apprenticeship; Civil Litigation Practice; Civil Matter Moot Court; Criminal } \\
\text { Proceedings Practice; Tax Dispute Practice; Patent Dispute Practice; Labor and } \\
\text { Social Security Dispute Practice; Administrative Litigation Practice; Medical } \\
\text { Criminal Disputes Practice; Public Interest Legal Aid Practice }\end{array}$ \\
\hline IU & $\begin{array}{l}\text { Feminism and Law Practice; IP Contract Practice; Traffic Law Practice; Minority } \\
\text { and Human Rights Practice; Civil Matter Practice; Family Dispute Practice; } \\
\text { Environment Law and Enforcement Practice; Criminal Matter Practice; Juvenile } \\
\text { Delinquency Theory and Practice; IP Litigation Practice; U.S. Patent Law and } \\
\text { Practice; Technology Licensing Practice; Administrative Litigation Practice; } \\
\text { Constitutional Litigation Theory and Practice; M\&A Theory and Practice; ADR } \\
\text { Practice; Bankruptcy Theory and Practice; Corporate Law Practice; Diplomacy and } \\
\text { International Law Practice; Copyright Litigation Practice; Franchise Law and } \\
\text { Practice; Computer Law and Practice; IT Venture Capital and Practice; Supply } \\
\text { Chain Management Practice }\end{array}$ \\
\hline CNNU & Legal Counseling Clinic; Labor Disputes Clinic; Farmers and Fisheries Dispute \\
\hline
\end{tabular}




\begin{tabular}{|c|c|}
\hline & $\begin{array}{l}\text { Clinic; Public Internet Human Right Clinic; Tax Disputes Practice; Civil Matters } \\
\text { Enforcement and Preservation Practice; Diverse Civil Matters Practice; Criminal } \\
\text { Law Practice; Environment Disputes Practice; Civil Proceedings Practice; Family } \\
\text { Proceedings Practice; Public Law Practice }\end{array}$ \\
\hline CBNU & $\begin{array}{l}\text { Finance Dispute Cases; Human Rights and Policy; Constitutional Law Drill: } \\
\text { Commercial Law Drill; Diverse Civil Law Drill; Diverse Administrative Law Drill; } \\
\text { Civil Proceedings Law Drill; Labor Law Drill; Criminal Law Drill; Company Law } \\
\text { Drill; Economic Law Drill; International Law Drill; Damage Claim Law Practice; } \\
\text { Administrative Litigation Practice; Labor Law Practice; Social Law Practice; } \\
\text { Criminal Proceedings Practice; Criminal Case Analysis; Civil Proceedings Practice; } \\
\text { IP Practice; International Business Transactions Practice; Preservation Litigation } \\
\text { Practice }\end{array}$ \\
\hline JNU & $\begin{array}{l}\text { International Investment Practice; International Contract Practice; Administrative } \\
\text { Litigation Practice; Criminal Private Practice; Family Disputes Practice; Civil } \\
\text { Matters Private Practice; IP Practice; Tax Litigation Practice; International } \\
\text { Litigation and Arbitration Practice }\end{array}$ \\
\hline CAU & $\begin{array}{l}\text { Accounting for Lawyers; English for Legal Practitioners; Legal Statistics and } \\
\text { Analysis; Legal Chinese; Psychology of Persuasion; English Legal Writing \& } \\
\text { Research; Civil Matters Private Practice 1; Criminal Matters Private Practice 1; } \\
\text { Civil Clinic; Criminal Clinic; Public Interest Clinic; Civil Matters Private Practice } \\
\text { 2; Criminal Matters Private Practice 2; Negotiation; International Commercial } \\
\text { Arbitration Practice; Criminal Evidence Law Practice; Economic Law Practice; } \\
\text { M\&A Practice }\end{array}$ \\
\hline $\mathrm{CNU}$ & $\begin{array}{l}\text { Civil Proceedings Practice; Civil Enforcement Practice; Civil Special Procedure } \\
\text { Practice; Civil Matters Private Practice; Diverse Criminal Matter Practice; } \\
\text { Economic Cooperation with North Korea and Dispute Resolution; International } \\
\text { Business Transactions and Corporate Litigation Practice; Corporate Litigation } \\
\text { Law and Practice; Administrative Proceedings Practice; Protection of Inventions } \\
\text { and Patent Practice; Patent Court and Litigation; Investment in China; IP } \\
\text { Infringement and Litigation; Trademark Law for Global Practitioners; } \\
\text { International Licensing and Negotiation; Patent Law for Global Practitioners }\end{array}$ \\
\hline CBU & $\begin{array}{l}\text { Practice of Compensation for Damages; Theories and Practice of Negotiable Instruments } \\
\text { Law; Theories and Practice ofLaw Related with Culture; Practice of Civil Procedure; Practice of } \\
\text { Real Estate Law; IP Judgment and Litigation; Practice of Commercial Contracts; } \\
\text { Practice of Patent Litigation }\end{array}$ \\
\hline HUFS & $\begin{array}{l}\text { Criminal Evidence Law and Fact Finding; Real Estate Registration Law; } \\
\text { International Negotiation; Criminal Defense Practice; Civil Matters Private } \\
\text { Practice; Secured Transaction Practice; International Financial Transaction } \\
\text { Practice; International IP Strategy Law and Practice; U.S. Law Practice; EU Law } \\
\text { Practice; Japanese Law Practice; Chinese Law Practice; Russian Law Practice; } \\
\text { India Law Practice; Latin American Law Practice; Middle Eastern Law Practice }\end{array}$ \\
\hline $\mathrm{HU}$ & $\begin{array}{l}\text { Practice of Civil Law; Practice of Public Law; Practice of Criminal Law; Practice } \\
\text { of Corporate Law; Practice of ADR }\end{array}$ \\
\hline
\end{tabular}


Table 3: JRTI Courses

\begin{tabular}{|l|l|}
\hline $\begin{array}{l}\text { Theory (First \& Second Sem.): Twelve } \\
\text { Courses }\end{array}$ & $\begin{array}{l}\text { Practice (First \& Second Sem.) Twenty-One } \\
\text { Credits }\end{array}$ \\
\hline $\begin{array}{l}\text { Real Estate Litigation (1); Preservation } \\
\text { Litigation (1); Investigation Process (1); } \\
\begin{array}{l}\text { Civil Enforcement (1); Damage Claims (1); } \\
\text { Legal English (1); Introduction to Common } \\
\text { Law (1); five electives (5) }\end{array}\end{array}$ & $\begin{array}{l}\text { Private Practice Training; Courthouse } \\
\text { Training; Public Prosecutor's Office } \\
\text { Training; On-the-job Training, [“OJT”] }\end{array}$ \\
\hline & \\
\hline & $\begin{array}{l}\text { Practice (Third \& Forth Sem.) Twenty-Six } \\
\text { Credits }\end{array}$ \\
\hline & $\begin{array}{l}\text { Private Practice Training; Courthouse } \\
\text { Training; Public Prosecutor's Office } \\
\text { Training; OJT }\end{array}$ \\
\hline
\end{tabular}

\section{B. Mandatory Versus Elective}

The Law School Law requires five mandatory practical courses: a) professional responsibility; b) legal research; c) legal document drafting; d) moot court; and e) an internship. ${ }^{32}$ With the exception of these five mandatory practice courses, the Law School Law leaves the curriculum open to each law school. The Guidelines, as a matter of fact, express a preference that more courses be elective. Thus, the Guidelines award ten points if the mandatory courses comprise less than 35 credits. ${ }^{33}$ As the number of mandatory credits increases, the score is reduced to as little as two points. On the other hand, the propriety of non-mandatory courses was also assessed on a ten-point scale. ${ }^{34}$ The number of elective courses was given a score of twelve to twenty points as the credit totals required for graduation ranged from one-hundred and ninety to two-hundred and thirty credits. ${ }^{35}$ Since the minimum number of credits is ninety, ${ }^{36}$ a student would have the option to choose fiftyfive elective credits out of the one hundred and ninety to two hundred and thirty credits. The

\footnotetext{
${ }^{32}$ Law School Law Enforcement Decree, Art. 13.

${ }^{33} I d$., Item 3.5.1.

${ }^{34} I d$., Item 3.5.2.

${ }^{35} I d$., Item 3.6.1.

${ }^{36}$ Law School Law, Art. 19, Para. 1 \& Law School Law Enforcement Decree, Art. 12, Para. 1.
} 
number of credits and the courses that law schools require to be taken are set forth below in

Table 4.

According to the plan of each law school, the number of mandatory courses ranges from twelve to thirty-nine. The contents of the additional mandatory courses are, however, similar in the sense that they are largely limited to contract, tort, property, civil and criminal procedure, and constitutional law in most law schools. Some law schools also added debtorcreditor law, commercial transactions, company laws, and administrative law courses. YLS is peculiar in requiring one basic theory course such as jurisprudence, philosophy, or sociology of law and one international law course.

Table 4: Number of Credit and Title of Mandatory Courses ${ }^{37}$

\begin{tabular}{|c|c|c|}
\hline Name of School & Credits & $\begin{array}{l}\text { Courses (In Addition to the Mandatory Five Practice } \\
\text { Courses) }\end{array}$ \\
\hline KNU & 24 & $\begin{array}{l}\text { General Provisions of Civil Law; Criminal Law I; Study on } \\
\text { Civil Rights; Obligations Law I; Criminal Procedure Law; } \\
\text { General Theory of Administrative Law; Civil Procedure Law I; } \\
\text { Corporate Law }\end{array}$ \\
\hline KKU & ND & ND \\
\hline KPNU & 14 & $\begin{array}{l}\text { Constitutional Law I; Civil Law I; Civil Law II; Criminal } \\
\text { Law I; IT Economy and Law }\end{array}$ \\
\hline KHU & & $\begin{array}{l}\text { Constitutional Law I; Civil Law I; Criminal Law I; } \\
\text { Constitutional Law II; Administrative Law; Civil Law II; } \\
\text { Civil Procedure; Criminal Procedure; Company Law }\end{array}$ \\
\hline KU & ND & $\begin{array}{l}\text { Civil Law I; Civil Law II; Civil Law III; Civil Procedure I; } \\
\text { Civil Procedure II; Constitutional Law I; Constitutional } \\
\text { Law II; Criminal Law I; Criminal Law II }\end{array}$ \\
\hline DAU & 26 & $\begin{array}{l}\text { Constitutional Law I; Constitutional Law II; Administrative } \\
\text { Law I; General Parts of Civil Code; Property; Obligations } \\
\text { Law I; Civil Procedure; Obligations Law II; Criminal Law } \\
\text { I; Criminal Law II; Criminal Procedure; Commercial } \\
\text { Transactions; Company Law; International Sale of Goods }\end{array}$ \\
\hline PNU & 39 & $\begin{array}{l}\text { General Parts of Civil Code; Obligations Law I; } \\
\text { Commercial Transactions; Constitutional Law I; General } \\
\text { Part of Criminal Law; Property; Obligation Law II; } \\
\text { Company Law; Constitutional Law II; Individual Part of } \\
\text { Criminal Law; Civil Procedure I; Administrative Law; } \\
\text { Criminal Procedure I }\end{array}$ \\
\hline
\end{tabular}

\footnotetext{
${ }^{37}$ For the English name of each law school, see supra note 5.
} 


\begin{tabular}{|c|c|c|}
\hline SU & 29 & $\begin{array}{l}\text { Public Law; Criminal Law; Civil Law; Contract; Civil } \\
\text { Procedure; Constitutional Law II; Administrative Law; } \\
\text { Torts; Company Law; Criminal Procedure }\end{array}$ \\
\hline SNU & ND & $\begin{array}{l}\text { Public Law I; Civil Law I; Criminal Law I; Civil Law II; } \\
\text { Civil Law III; Civil Procedure; Criminal Law II; Public } \\
\text { Law II; Public Law III }\end{array}$ \\
\hline UOS & 20 & $\begin{array}{l}\text { Constitutional Law I (Power Structure); General Part of } \\
\text { Civil Code; General Part of Obligation Law Chapter; } \\
\text { Criminal Law I; Contracts; Criminal Procedure; } \\
\text { Constitutional Law II (Basic Rights); Property; Basic } \\
\text { Commercial Code; Basic Administrative Law }\end{array}$ \\
\hline SKKU & ND & $\begin{array}{l}\text { Constitutional Law (Basic Rights); Constitutional Law II } \\
\text { (Power Structure); Contracts; Criminal Law; } \\
\text { Administrative Law; Civil Procedure; Torts; Criminal } \\
\text { Procedure; Company Law }\end{array}$ \\
\hline $\mathrm{AU}$ & 24 & $\begin{array}{l}\text { Constitutional Law Basic; Civil Law Basic; Criminal Law } \\
\text { Basic; Administrative Law Basic; Commercial Transaction; } \\
\text { Contract; Company Law Basic; Criminal Procedure Basic; } \\
\text { Criminal Procedure Basic; Public Law Dispute Resolution; } \\
\text { Civil Dispute Resolution; Criminal Dispute Resolution }\end{array}$ \\
\hline YLS & 34 & $\begin{array}{l}\text { Constitutional Law I; Constitutional Law II; Contract; } \\
\text { Property; Torts; Criminal Law II; Criminal Law II; } \\
\text { Criminal Procedure; Civil Procedure I; International Law; } \\
\text { Basic Theory }\end{array}$ \\
\hline YNU & 30 & $\begin{array}{l}\text { Constitutional Law Basic; General Part of Civil Code; } \\
\text { Contract; Property; General Part of Criminal Code; } \\
\text { Constitutional II (Basic Rights) General Part of } \\
\text { Administrative Law. Company Law; Civil Procedure I; } \\
\text { Criminal Procedure }\end{array}$ \\
\hline WU & 21 & $\begin{array}{l}\text { Constitutional Law; Civil Law; Criminal Law; } \\
\text { Administrative Law; Commercial Law; Civil Procedure; } \\
\text { Criminal Procedure }\end{array}$ \\
\hline EWU & 12 & $\begin{array}{l}\text { Basic Principles of Public Law; Criminal Law Basic; } \\
\text { Civil/Commercial Law Basic; Introduction to Civil and } \\
\text { Criminal Procedure }\end{array}$ \\
\hline IU & 26 & $\begin{array}{l}\text { Constitutional Law I; Criminal Law I; Civil Law I; } \\
\text { Administrative Law I; Criminal Procedure; Civil Law II; } \\
\text { Civil Procedure; Commercial Law }\end{array}$ \\
\hline CNNU & 29 & $\begin{array}{l}\text { Legal English; Civil Law Basic; Contract; General Part of } \\
\text { Criminal Law; Constitutional Law (Basic Rights); Property; } \\
\text { Civil Procedure; Individual Part of Criminal Law; } \\
\text { Administrative Law Basic; Business Forms }\end{array}$ \\
\hline CBNU & 30 & $\begin{array}{l}\text { Basic Rights; Constitutional Law Basic; Civil Law I; Civil } \\
\text { Law II; Civil Law III; Criminal Law I; Administrative Law } \\
\text { Basic; Company Law; Criminal Procedure; Civil Procedure }\end{array}$ \\
\hline JNU & 24 & $\begin{array}{l}\text { Constitutional Law; Civil Law; Criminal Law; } \\
\text { Administrative Law; Contract; Commercial Law; Civil } \\
\text { Procedure; Criminal Procedure }\end{array}$ \\
\hline CAU & 26 & Con Law I; Administrative Law I; Civil Law I; Civil Law \\
\hline
\end{tabular}




\begin{tabular}{|l|l|l|}
\hline & & $\begin{array}{l}\text { II; Criminal Law I; Constitutional Law II; Administrative } \\
\text { Law II; Civil Law III; Civil Law IV; Company Law I; Civil } \\
\text { Procedure I; Criminal Law II; Criminal Procedure; } \\
\text { Company II; Civil Procedure II }\end{array}$ \\
\hline CNU & 30 & $\begin{array}{l}\text { Intro to Civil Law; Contract; Criminal Law; Basic Rights; } \\
\text { Administrative Law I; Property; Torts; Civil Procedure; } \\
\text { Company; Power Structure; Criminal Law II }\end{array}$ \\
\hline HUU & 21 & $\begin{array}{l}\text { General Theories of Constitutional Law; General Theories } \\
\text { of Civil Law; General Theories of Criminal Law; General } \\
\text { Theories of Civil Procedure; General Theories of } \\
\text { Commercial Law; General Theories of Administrative Law; } \\
\text { General Theories of Criminal Procedure }\end{array}$ \\
\hline HU & ND & $\begin{array}{l}\text { Constitutional Law; Criminal Law General Part; Contract; } \\
\text { Torts; Commercial Law; Administrative Law; Criminal } \\
\text { Procedure; Property; Civil Procedure I; Company Law }\end{array}$ \\
\hline ND & $\begin{array}{l}\text { Civil Law I(Property); Civil Law I(Contracts); Criminal } \\
\text { Law I; Constitutional Law I(Power Structure); Civil Law } \\
\text { III(Torts); Civil Law IV(Security Interest); Administrative } \\
\text { Law; Civil Procedure }\end{array}$ \\
\hline
\end{tabular}

\section{Local Versus Global}

Although the need to globalize legal education in Korea has been increasingly stressed, the Law School Law does not specify such requirements in writing. The Guidelines, however, give ten points to applicants if five or more novel courses are promised to be offered. Novel courses may be used to foster global perspectives by comparing legal systems or by applying different perspectives from other disciplines to the legal system. Global courses are set forth below in table 5. Ten points are also awarded if twenty or more credits are to be taught in English. ${ }^{38}$

According to the plans submitted, the number of credits taught in foreign languages ranges from seven to twenty-six. ${ }^{39}$ The number of faculty members who can speak English

\footnotetext{
${ }^{38}$ Law School Law Enforcement Decree, Art. 13, Items 3.6.3 \& 3.6.4.

${ }^{39}$ The details are as follows: KNU (seven credits in English); KU (twenty-one credits in English); DAU (ten credits in English/three credits in Japanese/two credits in Chinese); SU (nineteen credits in English/one credit in Japanese); SU (fifteen credits in English/one credit in Chinese); YLS (twenty-six credits in English/one credit in Chinese); WU (eighteen credits in English); and CBU (eleven credits in English).
} 
or other foreign languages ranges from three to ten. ${ }^{40}$ Each law school is required to specialize in one or more particular sectors. While the most popular specialty areas are corporate, science and public interest, global legal matters are designated by four law schools. ${ }^{41}$ Despite the sparse availability of foreign faculty and foreign language courses, the diversity in terms of disciplines and geographical areas is immense, as demonstrated in Table 5. Economics, finance, political science, sociology, and medical science are to be taught while laws of many foreign jurisdictions such as Russia, Latin America, the Middle East, and South East Asia are covered.

Certain graduate schools offer so-called joint degree programs with foreign law schools. For example, Yonsei graduate school offers LL.M. programs in conjunction with Washington University School of Law and American University College of Law if one semester is completed there. While major universities are now converted into three-year law schools, their futures are not quite clear as the memoranda of understanding [MOUs] with these law schools fail to cover the incoming graduate law school students. Although these arrangements can probably be expanded to the new law school students with ease, it is doubtful whether these students will wish to defer bar exams for one year to get an LL.M. from these American law schools. Most recently, Korea University launched an executive LL.M. program for junior lawyers, including newly graduated law school students in the near future. ${ }^{42}$ As for law school students, while some U.S. law schools are promoting joint degree programs, YLS signed an MOU with the University of Washington School of Law, according to which, law school students can get LL.M. degrees by attending two quarters and acquiring

\footnotetext{
${ }^{40}$ Available at websites of each law school. As for the range of foreign students, see Soon-Hyuk Lee, Keudaeneun 20dae-SKY-gangnam ch'ulshin [They are all in their 20's, SKY University Graduates, \& Residing in Gangnam District], HANGYERAE ONLINE, http://h21.hani.co.kr/arti/cover/cover_genera1/24406.html.

${ }^{41}$ Dae-Heon Bae, A Study on Specialized Education Program of Law School in Korea, 27 KoREA INDUS. PROP. L. J.1, 8 (2008).

${ }^{42}$ See Korea University Law School, Koryeodae-Wash U Executive LLM kwajeong sogaemun [Korea University Law School-Wash U LLM Program Introduction], http://www.korealawschool.com/korea.asp?ai=6.
} 
thirty credits. ${ }^{43}$ If students choose this option, they can sit for the U.S. bar exam before they graduate from their Korean law schools. Although U.S. qualification may still have some significance relating to the individual's intellectual capability as a lawyer, its practical importance would be faded out quickly. However, a basic understanding of the U.S. legal system would definitely help the students to understand such facets of the Korean legal system as corporate and capital markets regulations. YLS also signed an MOU under which law school students can attend summer programs at Indiana University‘s Maurer School of Law beginning in summer 2010, which few students are likely to be interested in. ${ }^{44}$ As an alternative, some law schools have contrived to conduct summer sessions with foreign faculty members. One example is SNU's summer program held in 2009 with a focus on U.S. legal systems.

Table 5: Global Courses

\begin{tabular}{|l|l|}
\hline Diverse Legal Systems & Diverse Disciplines \\
\hline 1. U.S. Law & 1. Accounting, International Business, \\
U.S. Business Law; U.S. Environmental Law & Finance, International Trade \& \\
\& Regulation; U.S. Uniform Commercial & Environment \\
Code; U.S. Contract Law; Private Law in & Corporate Accounting; Law and \\
Common Law Jurisdictions; Private Law in & Accounting; Accounting Auditing; \\
Anglo-American Legal Tradition; Public Law & International Transportation; International \\
in Anglo-American Legal Tradition; U.S. & Supply Chains; International Business \\
Securities Law; U.S. Civil Procedure; & Transactions in Global Era; International \\
American Torts Law; The Constitution of the & Business Management; Legal Environment \\
United States; American Criminal Law \& & of Business; Management Strategy; \\
Procedure; U.S. IP Law; U.S. Negotiable & Internal Control and Compliance; Seminar \\
Instruments; the Judicial System of the & on Comparative Anglo-American \\
United States; American Civil Procedure & Corporations; Corporate Governance and \\
Rules \& Practice; U.S. Patent Law and & Corporate Social Responsibility; \\
Practice; U.S. Evidence Law; UCC; U.S. & Enterprise Forms; M\&A Case Study; \\
Real Estate Law & M\&A and Taxation; Capital Markets; \\
& Finance \& Tax; Financial Management; \\
\hline
\end{tabular}

${ }^{43}$ Tae-Gyun Kim, U.S. Bar Exams Accessible from Yonsei Law School, YonHAP OnLINE, Jan. 29, 2010 http://www.yonhapnews.co.kr/society/2010/01/29/0702000000AKR20100129079400004.HTML?template=208 9.

${ }^{44}$ See Indiana Univ. School of Law, Application Instructions for the IU Maurer Summer Law Institute, available at http://law.indiana.edu/degrees/graduate/doc/SummerLawApplication.pdf. 


\begin{tabular}{|c|c|}
\hline $\begin{array}{l}\text { 7. Chinese Law } \\
\text { Chinese Economic Law; Chinese External } \\
\text { Economic Law; Chinese IP Law; Chinese } \\
\text { Civil Procedural Law; Chinese Trade Law; } \\
\text { Chinese Legal History; Chinese Judicial } \\
\text { System; Chinese Contract Law; Chinese } \\
\text { Competition Law; Chinese Foreign } \\
\text { Investment Law; Chinese Securities } \\
\text { Regulation; Chinese Commercial Transaction } \\
\text { Law; Chinese Conflict Law; Chinese } \\
\text { Enterprise Law; Chinese Legal Theory; } \\
\text { Chinese Taxation General Law and Practice; } \\
\text { Chinese Bankruptcy Law; Chinese Taxation } \\
\text { Special Law and Practice; Chinese Corporate } \\
\text { Law; Chinese Land Law } \\
\text { 8. Japanese Law } \\
\text { Japanese Administrative Law and Public } \\
\text { Policy; Japanese Contract Law; Modern } \\
\text { Japanese Law; Japanese Financial Products } \\
\text { Transaction Law; Japanese Society Study; } \\
\text { Japanese Patent Law } \\
\text { 9. North Korean Law } \\
\text { North Korea Economic Law; North Korea } \\
\text { Law in the International Context; North } \\
\text { Korea Criminal Law; Investment Law in } \\
\text { North Korea; Unification Law Seminar } \\
\text { 10. Middle Eastern Law }\end{array}$ & 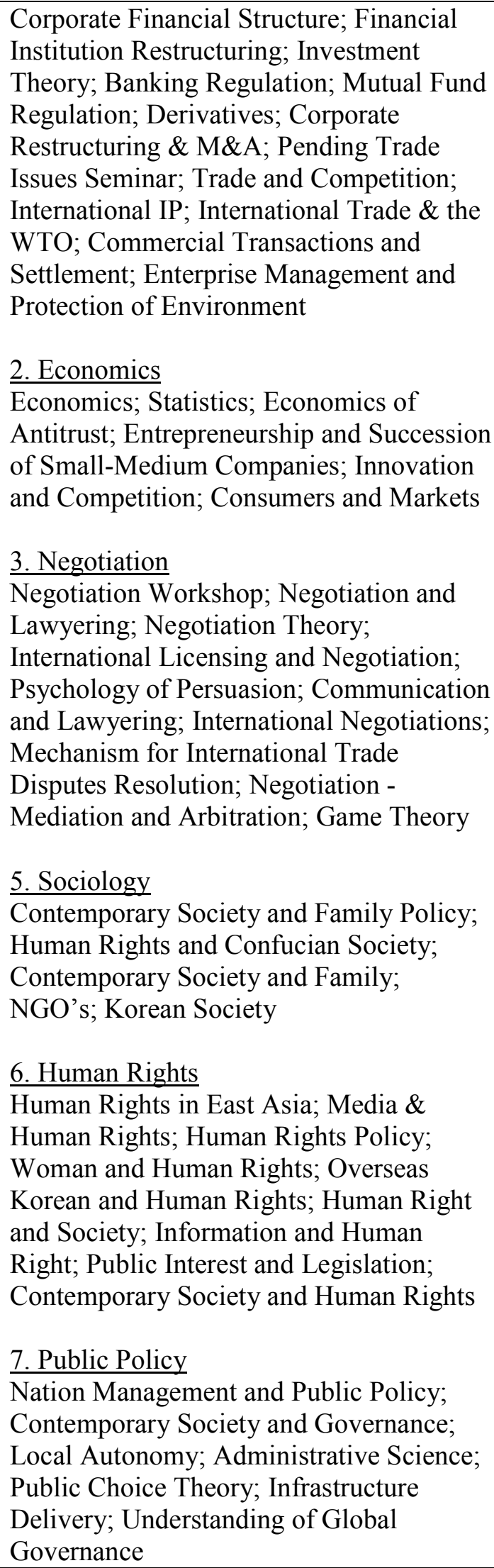 \\
\hline
\end{tabular}


Northeast Asian Environment Law; Northeast

Asian Economic Law

12. South East Asian Law

\section{Politics}

Politics and Political Science; Democracy and World Order; International Relations:

Theory and Practice

9. Science \& Environment

Environment; Energy and Climate Change; Invention and Patent Practice; Law and Ecology

10. Medical Science

Ethics \& Medical Science; Inspection of Medical Practice

III. ReAsons for More Practical, Global, Elective AND Non-Litigation Courses

The education system in Korea has, as its stated goal, the aim of helping all citizens to lead humane lives and contribute to the development of a democratic nation and the common wealth by building character, furnishing autonomous living capability, and teaching the traits of democratic citizens under the idea of human beings doing good towards others. ${ }^{45}$ The Basic Education Law classifies education into three groups: obligatory, school, and social. ${ }^{46}$ The Higher Education Law also describes the goals of universities as providing a place for researching and teaching the theories and practical skills that are necessary for the development of the nation and human society in general. ${ }^{47}$ Universities granted charters from the government may also establish professional graduate schools to research and apply practical theories to produce qualified professionals. ${ }^{48}$ The Law School Law describes the tasks of law school education as production of legal professionals that will provide high quality legal services in response to the diverse demands and requests of the citizens, equipped with (a) professional ethics based on rich cultures, a deep understanding of human beings and societies and a value system based on freedom, liberty and justice, and (b) the

\footnotetext{
${ }^{45}$ Kyoyuk kibon beop [Basic Education Law], Law No. 8915 of 2008, Art. 2.

${ }^{46}$ Id., Arts. 8, 9 and 10.

${ }^{47}$ Koteung kyoyuk beop [Higher Education Law], Law No. 8988 of 2008, Art. 28.

${ }^{48}$ Id., Art. 29-2, Para. 1, Item 2.
} 
knowledge and capability to resolve complicated legal disputes professionally and

effectively. ${ }^{49}$ Although the purposes of legal education under the foregoing statutes are worded extremely broadly, ${ }^{50}$ it is simple and clear that the goal of legal education is to produce competent lawyers to analyze, prevent, and resolve social problems as professionals. $^{51}$

\section{A. Reasons for Practical Courses}

In the past, law colleges were responsible for preparing students for the bar examination, while the JRTI was responsible for practical training. The practical training at the JRTI, however, was meant to produce government bureaucrats such as judges and public prosecutors, although this objective has been diversified a little bit since 1995. As the law school system is instituted, two thousand law school graduates will take the new bar examination in 2012 , most of whom will start their private practice without any training at the JRTI. Thus, the responsibility to provide the practical training that would have been conducted at the JRTI must ultimately be shifted to law schools. However, the principal part of the JRTI training comprised of drafting court judgments and letters of indictment would be assigned to courts and public prosecutor's office. ${ }^{52}$

${ }^{49}$ Law School Law, Art. 2.

${ }^{50}$ The Carnegie Foundation proposed six tasks to reach the three goals of knowledge, skills, and attitude in legal education: 1) developing in students fundamental knowledge and skills, especially an academic knowledge base and research abilities; 2) providing students with the capacity to engage in complex practice; 3 ) enabling students to learn to make judgments under uncertain conditions; 4) teaching students how to learn from experience; 5) introducing students to the disciplines of creating and participating in a responsible and effective professional community; and 6) forming students able and willing to join enterprise of public service.

CARNEGIE REPORT, supra note 14, at 22.

${ }^{51}$ For a critical view about the abrupt departure of the new system from historic heritage and wisdom in Japan, see Koichiro Fujikura, Reform of Legal Education in Japan: The Creation of Law Schools Without a Professional Sense of Mission, 75 TUL. L. REV. 941 (2001). But see Mark Levin, Legal Education for the Next Generation: Ideas from America, 1 AsIA-PAC. L. \& POL'Y J. 1, 13-14 (2000). Levin stresses the fact that the feedback from the graduate students who took negotiation skills courses was uniformly positive.

${ }^{52}$ On Sep. 22, 2008, JRTI organized a tour for practice professors. During the tour, Young-Soo Kang, J., distributed a 44-page pamphlet entitled JRTI Education and Law Schools. See supra note 22. Most of the courses except for moot courts do not appear to be relevant to the practical education at law schools. For a comparison with Japanese guidelines, see Chang Rok Kim, Symposium 1: Practical Perspectives on Korean 
Aside from the impending needs arising from such institutional changes, expanding the philosophical perspectives of lawyers is a more important reason for practical courses to be offered at law schools. Lawyers used to be trained as functional bureaucrats in Korea, ${ }^{53}$ while lawyers at the new law schools are to be trained as legal professionals. William Sullivan posits that all professional schools must train their students in "three apprenticeships:” intellectual capability; skill; and values and ideals ${ }^{.54}$ In essence, law schools must train their students to have legal skills and ethics of everyday law practice. Skills and ethics can be more effectively taught in a quasi-real situation by practitioners with actual work experience. This is why the Law School Law requires one fifth of faculty members to have five or more years of experience practicing. ${ }^{55}$ In addition, five practical courses (professional responsibility, legal research, legal document drafting, and an externship) are mandatory for students. ${ }^{56}$ In this light, it is interesting that the apex of practicality, U.S. law schools, have been recommended to join "lawyering," professionalism, and legal analysis from the start of the law school curriculum. ${ }^{57}$

Law Schools; Education of Theories and Education of Practices at Law School, 35 KOREAN LAW AND SOCIETY 9, 12 (2008).

${ }^{53}$ They may be called the "power elites of Korean society." For those who got legal education as the "power elites of Japanese society," see Setsuo Miyazawa \& Hiroshi Otsuka, Legal Education and the Reproduction of the Elite in Japan, 1 AsIAN-PAC. L. \& POL'Y J. 1, 15 (2000). The elitism is so skewed in favor of University of Tokyo that reverse discrimination might be needed.

${ }^{54}$ William M. SUllivan, Work AND INTEGRITY: THE CRisis AND Promise OF Professionalism IN AMERICA 207-16 (2d ed. 2005).

${ }^{55}$ Law School Law, Art. 16, Para 2.

${ }^{56}$ Law School Law Enforcement Decree, Art. 13, Para.1.

${ }^{57}$ See William M. Sullivan ET AL., The CARnEgie Foundation For the AdVANCEMENT OF TEAChing, Educating LaWyers: Preparation for the Professon of LAW: Summary 9 (2007), available at http://www.carnegiefoundation.org/sites/default/files/publications/elibrary_pdf_632.pdf. During our interview with him at YLS, Prof. Joseph Hoffman of the Indiana University Maurer School of Law indicated that legal ethics course had been made into a $1 \mathrm{~L}$ course at Maurer. 


\section{B. Reasons for Global Courses}

As the Korean economy has globalized, many have recognized that globalization is not only an economic issue but also a challenging legal issue. ${ }^{58}$ How to coordinate the diverse interests of people in the global economy is one example of the serious legal issues arising out of globalization. ${ }^{59}$ To overcome this challenge, legal professionals, like other professionals, should be able, at the very least, to understand the legal systems of other countries and cultures. Such an understanding, of course, would put local and parochial legal systems into context such that lawyers may reach a better understanding of jus gentium. In addition, Korea ought to spread its experience of reaching economic goals by means of the legal principles and tools to the developing countries. ${ }^{60}$

In practice, lawyers have been requested to advise the public on international legal matters and resolve trans-border disputes. While in the international arena, lawyers with an Anglo-Saxon educational background still dominate, largely because of their clients' economic standing and their language skills. Korean legal education should be able to produce lawyers able to communicate, coordinate, and cooperate with foreign lawyers in the context of many aspects of the Korean economy. Furthermore, the Korean economic presence has extended to many parts of the world via globalization and the Korean diaspora; lawyers will have to provide the necessary legal assistance to address the legal issues arising from this expansion on the spot. Thus, although no specific global courses are required by

\footnotetext{
${ }^{58}$ See Winston P. Nagan, FRSA \& Danie Visser, The Global Challenge to Legal Education: Training Lawyers for a New Paradigm of Economic, Political and Legal-Cultural Expectations in the $21^{\text {st }}$ Century, 11 ILSA J. INT'L \& COMP. L. 9 (2004) (arguing that the practice of law will become increasingly globalized throughout the twenty-first century).

${ }^{59}$ See Kal Raustiala \& Alfred C. Aman, Jr., The Democracy Deficit: Taming Globalization through Law Reform, 55 J. LEGAL EDUC. 446, 447 (2005) (“[D]emocratic decision-making. . is threatened. ... .”).

${ }^{60} \mathrm{KDI}$, the Center for Economic Cooperation, has been engaged in international development assistance activities as a government agency. In the future, Korean law schools ought to play a role in helping developing countries in establishing rule of law through programs like Indiana University's Center for International Education and Development Assistance and various initiatives at Adilet Law School in Almaty, Kazakstan. See generally Roger Burridge, Six Propositions for Legal Education in Local and Global Development, 55 J. LEGAL EdUC. 488 (2005). See also Simon Chesterman, The Globalization of Legal Education, 2008 SingAPORE J. LEGAL STUDIES 58.
} 
the Law School Law, many courses are encouraged to be taught in English and many foreign law courses to be added to the curricula. "Global" does not have to be narrowed to a geographical concept, but should be expanded to diverse perspectives from different disciplines. As such, law schools should be more open to the possibility of adopting and incorporating the diverse perspectives from liberal arts and social science.

\section{Reasons for Elective Courses}

Legal education by law schools should play a much broader role than simply producing public officials in the judicial branch or public prosecutor's office. Although such education would be largely limited to the training of professional lawyers (rather than judicial bureaucrats), these graduates will not have to practice law solely at private firms, but can also work for the executive or legislative branches, international institutions, and NGOs. They can work not only at private enterprises but also for public interest organizations. Thus, mandatory courses should be limited to the core of the system while law schools should be granted wider freedom. Thus, the Law School Law, requiring ninety credits, including five mandatory practical courses, gives chartered law schools complete freedom to design courses. $^{61}$

\section{Reasons for Non-Litigation Courses}

When the principal purpose of legal education is to ensure students who pass the national bar exams are appointed as career judges or public prosecutors, it is natural that all courses should be about adjudication. If, however, the role of lawyers has been expanded from the so-called "beobjoin" 62 to working within or advising private companies, drafting statutes or contracts, negotiating deals or settlements, and even protecting union organizers or

\footnotetext{
${ }^{61}$ For an examination of the lack of diversity in Japan, see Dan Rosen, Schooling Lawyers, 2 AsIANPAC. L. AND POL'Y J. 66 (2001).

${ }^{62}$ The meaning or origin of this term is not clear, but, it usually refers to lawyers as public officials as judges or public prosecutors.
} 
the human rights of the handicapped or immigrants from North Korea or other less developed countries in Asia, courses at law schools should be expanded substantially beyond role playing in a traditional courtroom. It is not always clear that certain courses teach only dispute resolution while certain others are teach only dispute prevention. Nonetheless, civil and criminal laws and procedures are just starting points for all legal issues, so more diverse courses addressing legal problems before they reach an official or institutional tribunal should be created. ${ }^{63}$ Especially as Korean politics reach a more mature level of development, legal drafting for legislation and policy studies should play ever more important roles at law school curricula.

\section{PLANS FOR IMPLEMENTATION}

\section{A. Practical Courses}

\section{i. $\quad$ Number and Content of Classroom Practical Courses}

The availability of practical courses is currently too limited, and the contents of these courses are too unbalanced, to produce effective lawyers capable of solving legal problems. At YLS, only twelve interdisciplinary courses fitting the description of "applied practical courses" are to be administered. They include public law application, civil law application, criminal law application, commercial law application, public interest disputes, civil procedure disputes, criminal procedure practice, international business contract practice, law and practice of trans-border flow of goods/technology/capital, corporate compliance programs, finance and tax practice, and intellectual property licensing practice. If the "application" courses are similar to case studies at the senior year of law colleges, only eight courses are

\footnotetext{
${ }^{63}$ For the role of non-litigators in Japan, see George Schuhmann, Beyond Litigation: Legal Education Reform in Japan and What Japan's New Lawyers Will Do, 13 U. MiAmi INT'L \& COMP. L. REV. 475, 499 (2006). Reduction of information asymmetries, risk management, and verification costs are listed as the addedvalue of transactional lawyers. Their value as reputational intermediaries, gatekeepers and option brokers is also listed.
} 
truly for practical training, which seems far too low to meet the goal of instituting a law school system. ${ }^{64}$

YLS will offer seven clusters (public, civil, commercial, economics, science, international, and basic), with each cluster offering between three and seven courses. ${ }^{65}$ If each student takes one major and one minor cluster as his or her major, thirty credits for his or her second year would suffice to fulfill their requirements. In their third year, if students can concentrate their courses on in-depth courses in one major cluster (so-called "capstone" courses), one semester would be enough. Thus, the final semester in their third year could be allocated completely to applied practice courses. If this were the case, the desirable ratio of theory courses vis-à-vis practice courses could be five as a rule of thumb. Unlike U.S. legal practice, which is extremely specialized, the Korean legal community is still composed mainly of generalists. As law practice is professionalized, however, more specialization is desirable. Thus, fledgling new lawyers should be furnished with legal skills in a specific area to survive in the future.

Multiple and redundant courses should be substantially restructured in line with the specific tracks of disciplines. In fact, considering the size of the student body, the number of courses seems inappropriate. As the 2010 spring semester starts, most of the courses will not be open to the students. For example, YLS's spring term courses are limited to less than twenty courses. The same phenomenon will happen in most law schools and will continue

\footnotetext{
${ }^{64}$ It is not clear why application courses are added to the second or third-year curricula if the core courses are to be taught through case-centered methods at graduate school level. Almost everyone seems to agree that combination of the case method, clinical courses and externships is the right way, but the issue as of now seems to be how to implement this. If all the basic courses of the first year are case studies and comparative studies, no additional exercise class or comparative class would have to be offered as part of the law school curriculum. See Young-Cheol Kim, The Importance of Practice in the Legal Education, 47 SEOUL LAW REVIEW 4, 147 (2006).

${ }^{65}$ For the initial drawing of the curriculum at YLS, see Sang Yoon Lee, Sang Yong Kim, Sung Tae Kim, Sang Kee Park \& Young Hur, Part I: Plan and Methodology for Law Schools (Courses, Curriculum \& Pedagogy), 9 Yonsei LAW ReVIEW 335 (2000). For later phase discussions, see Hyun-Yoon Shin, Kil-Jun Park \& Jeong-Oh Kim, A Study on Improving Teaching System of Legal Education, 13 YONSEI LAW REVIEW 87 (2003).
} 
for the next three semesters, until fall 2011, which seems to be a normal and desirable endpoint for such a limitation.

The average ratio of theory courses vis-à-vis practical courses in many law schools exceeds five or more. In order to make law schools efficient institutions for producing lawyers with professionalism, the ratio should be reduced substantially so that the third year education program is more focused on practice. In an ideal situation, the ratio between theory and practice classes during the second and third years of law schools should be two to one so that the final year at law schools will be spent gaining practical skills with the guidance of the law schools' teachers.

In terms of content, the practice courses that used to be part of the senior year of undergraduate law colleges should be scrapped, highlighting instead more frequently disputed areas of the law. Furthermore, while litigation and arbitration are still, and will remain, a major part of new lawyers' practice, legislation and planning courses should be more widely offered. In that sense, the current plan also should be substantially redesigned and restructured. The list of examples of applied practical courses for each cluster at YLS is listed in Table 6.

Table 6: Desirable Basic Practice Courses for YLS

\begin{tabular}{|l|l|}
\hline Civil & $\begin{array}{l}\text { Real Estate Litigation, Redevelopment Litigation; Injunction and Attachment } \\
\text { Practice; Court Auction Practice }\end{array}$ \\
\hline Criminal & $\begin{array}{l}\text { Corporate Compliance Programs; White Collar Crime Investigation; Finance } \\
\text { Crime Investigation; Computer Crimes Investigation }\end{array}$ \\
\hline Commercial & $\begin{array}{l}\text { Corporate Litigation; Corporate Governance Design; International Finance } \\
\text { Documentation; M\&A Practice; Deal Negotiations }\end{array}$ \\
\hline Public & Legislation Practice; ${ }^{66}$ Constitutional Litigation; Legal Audit Practice \\
\hline Economic & $\begin{array}{l}\text { Public Private Partnership Consignment; Antitrust Litigation; Tax Litigation, } \\
\text { Derivatives and Tax; Tax Havens and Planning; Tax Audit Practice }\end{array}$ \\
\hline Science & $\begin{array}{l}\text { License Negotiation Practice; IP Litigation; International Patent Disputes; } \\
\text { Natural Resource Development Contract Practice; International Energy }\end{array}$ \\
\hline
\end{tabular}

${ }^{66}$ For the U.S. experience and a proposal for a legislation course as part of the first-year of law school, see Ethan J. Leib, Adding Legislation Courses to the First-Year Curriculum, 58 J. LEGAL EdUC. 166 (2008). 
Development Disputes

International $\quad$ WTO Disputes; International Arbitration Practice; Negotiation Practice

\section{ii. Practical Courses Outside the Classroom}

Practice cannot be taught only in the classroom. ${ }^{67}$ More diverse education methods devised and implemented by foreign law schools should be tested in Korea despite the uncertainty of their final outcome. Certain law schools are touting their plans to run legal clinics without a clear understanding of the legal issues such clinics entail. Although clinic courses have proven to be an excellent way of practice training in the United States, and many law schools are fierce advocates of clinical education, their future at Korean law schools is uncertain at best due to the lack of detailed consideration of the specifics of running them. First, if running a clinic falls within the meaning of practicing law, which is a reasonable interpretation, there is no way for law schools to carry on clinic programs because as of now no faculty members are allowed to practice law. ${ }^{68}$ Second, should the Law School Law be revised to permit law faculty members to run clinics, it is not clear what kind of legal issues are to be resolved at legal clinics. They may be limited to poor and meager individuals and enterprises, such as small claims, family matters, landlord-tenant issues, petty individual investors or small to medium sized enterprises. They may be expanded to include high-stake public interest legal issues, such as constitutionality cases, freedom of speech cases, and criminal cases. They may include university-related issues such as various contract, intellectual property, and transactional matters. All these details should be decided in 2010 while the first graduate law students are in their second year. Otherwise, no clinics will be launched in 2011, when the first class enters its third year. I believe there would be no reason to put limits on the subject matter of legal clinics. Legal clinics can fill the role of public

\footnotetext{
${ }^{67}$ Due to the limited financial resources of law schools and the peculiar exclusivity of the academic community, it is not easy to invite guest lecturers to the classroom in Korea. This is largely an internal problem of law schools, which will be improved in time.

${ }^{68}$ See infra Part IV.A.3.
} 
interest law firms, which are almost non-existent in Korea, by helping members of the public with limited financial resources and addressing the socially-sensitive issues that no established law firms dare address. Legal clinics can help the Korean legal community to revisit conflict of interest issues, which is an uncommon occurrence due to the oligopoly currently existing in the legal industry.

Practical training should consist of externships at outside institutions or law firms as well as clinics. At YLS, like most Korean law schools, one credit will be awarded for a one hundred and twenty-hour internship lasting three weeks at one of sixty-one or more outside institutions. However, most of them signed the same MOUs with all law schools. They might be too indifferent to the needs of law school students or too short-sighted to train them with long term goals in mind. In the summer of 2009, SNU hurriedly implemented two-week pilot programs with major law firms. This caused every law school to rush to implement similar programs for student internships in the winter of 2010, which have now generally lasted for one or two weeks. In addition, some government agencies which desire more public attention, such as the Constitutional Court and the Ministry of Government Legislation, accepted some law school students. However, they all seem to be too short and too ad-hoc. For the winter of 2010, law schools are running a hodgepodge of diverse, sometimes superficial, internship programs with law firms and agencies.

Finally, in January 2010 the Korean courts made public their plan to run apprentice programs in the summer of 2010 with a limited number of law school students. ${ }^{69}$ As sitting on one of the Korean courts is still regarded as the ultimate goal and the most successful position of the legal profession by many lawyers, their program set the standard. The program will last at least eight hours per day for two weeks by district courts. The number of

\footnotetext{
${ }^{69}$ In-Ha Ryu, In-Ha, Roseukul saeng 7weol but'eo beopweonsa shilmu suseup [Law School Students to Train at Court from July], BEOPRYUL SHINMUN, Jan. 18, 2010, available at http://www.lawtimes.co.kr/LawNews/News/NewsContents.aspx?kind=AA01\&serial=50998\&page=1.
} 
students and details are yet to be finalized. However, the focus will be on judicial proceedings. The Ministry of Justice, including the Public Prosecutor's Office, is expected to announce its own internship program in the near future for the summer of 2010. If so, Korean government agencies, private enterprises, and law firms will plan their own internship programs at least for the winter of 2011 when second year students have achieved in-depth legal analytical skills in their chosen areas.

\section{iii. Instructors for Practical Courses}

Although more than one-fifth of faculty members have five or more years of practice experience, the teaching of legal skills at law schools is not always effective for several reasons. ${ }^{70}$ First, the faculty for practical courses is not allowed to practice law if they want to be considered full time faculty members. ${ }^{71}$ Adjunct professorship is not prohibited, but the allowable weekly teaching hours for adjuncts shall be divided by nine in determining the size of the faculty to be maintained. Furthermore, as many law schools have maintained internal policies of not appointing adjunct professorship, their numbers are few. Faculty members with practice experience will soon lose their expertise and no new blood will come in. Second, while practicing law is one thing, teaching practice is another. Being a good teacher requires a different skill-set from that of a good practitioner, whether it be a judge who has sat at the bench for years, a public prosecutor experienced in investigating and prosecuting criminals, or a private practitioner who has extensive experience advising clients. Thus, licensed lawyers should continue to practice law while teaching at law schools. Yet, they would be forced to opt out the practice if they want to survive as teachers in the academia. To the extent their competence permits, they should be allowed to maintain contact with the

\footnotetext{
${ }^{70}$ Even in the United States, where clinic courses are a well-settled part of the curriculum, there seems to be some schism between the traditional scholarship and the goal of clinicians and activities. See Douglas L. Colbert, Broadening Scholarship: Embracing Law Reform and Justice, 52 J. LEG. EDUC. 540 (Dec. 2002).

${ }^{71}$ Guidelines, Item 4.3.1 requires that a filing with local bar associations that one will not continue practicing law be submitted. See also Law School Law Enforcement Decree, Art. 9, Para. 3.
} 
market. It would be good for law schools as well as the practice world. Law schools can get fresh input from the practicing world while the practicing world can get theoretical perspectives from the academic world. Furthermore, law schools should be more open to the possibility of secondments from the courts and public prosecutor's offices, both institutions which are somewhat anxious to maintain their influence over legal education. While they can work as a mouthpiece for courts and public prosecutor's office, they can also be a good channel of to the institutional clients of legal education.

\section{iv. Role Playing Argument}

Some argue that law schools should teach basic legal skills such as legal research and writing. Further training should be done by professional groups such as law firms, the judicial branch, the public prosecutor's office, the legislature, private enterprises, government agencies, NGO's, and international agencies, among others. ${ }^{72}$ This view might be based on the observation of reality that law schools need more time to accumulate practical experience educating students. However, more applied practical courses should be open to third year students assuming that the restrictions on law practice of faculty members are lifted. It is true that no clear distinction between theory courses and practice courses can be drawn. Even socalled theory courses cannot be taught without referring to actual cases, contract language and disputes. Since legal theories and content are the basis for legal analysis and skill, it makes sense that more focus should be put on legal theories and content. ${ }^{73}$ Since, however, more than one-fifth of faculty members have relocated themselves to academia from public or private practice, it would bring more value to law schools if they would be able to share

\footnotetext{
${ }^{72}$ See Jong Cheol Kim, Curriculum \& Pedagogy at Law Schools, in LAW SCHOOL AND LEGAL EDUCATION 96 (Kun-Shik Kim et al. eds. 2008). See also Jong Cheol Kim, Symposium 1: Practical Perspectives on Korean Law Schools: Legal Education as Liberal Arts and Sciences in the Era of Korean Law School System, 35 KoREAN LAW AND SocIETY 27, 31 (2008).

${ }^{73}$ For the unclear status of SNU, see Hong Sik Cho, A Blueprint for SNU Law School, 45 SEOUL LAW REVIEW 2, 35-42 (2004).
} 
their experience with the students. ${ }^{74}$ Drawing on these internal resources will be much more effective than relying on MOU's signed with many outside institutions competitively and without any serious deliberation. In addition, clinics should stand as a major piece of the curriculum to be planned with more effort and care. ${ }^{75}$ In addition, international moot court and writing competitions will have to be carefully weighed on and integrated as part of their curriculum. $^{76}$

\section{B. Elective Courses}

Because law school graduates are expected to pursue a variety of legal careers, the scope of mandatory legal education should be limited to the core of the legal system. As such, at YLS, twenty-eight credits in mandatory theory courses and six in mandatory practice courses are all required in the first year. In contrast to the mandatory thirty-four credits, three hundred and eighty-three credits from one hundred and forty-seven courses are electives. As mentioned earlier, except for one basic and one international course to be taken, fifty of ninety credits that would be required for graduation will be chosen from six clusters.

Forty credits might be enough to understand the core of the Korean legal system. Because, however, the Korean legal system is from the Roman-German-Pandekten system, the reshuffling of the contract/tort/property classes will not be easy for the time being. For this reason, debtor-creditor law and administrative law should also be mandatory. If so,

\footnotetext{
${ }^{74}$ Why so many private practicing lawyers or public officials moved to academia is a question which will require more research.

${ }^{75}$ For the experience of Japan, see Peter A. Joy, Shigeo Miyagawa, Takao Suami \& Charles D. Weisselberg, Building Clinical Legal Education Programs in a Country Without a Tradition of Graduate Professional Legal Education: Japan Educational Reform As a Case Study, 13 CLINICAL L. REV. 417 (2006). See also Elliot S.Milstein, Teaching Professional Values Though Clinical Legal Education, 22 RITSUMEIKAN LAW REVIEW 111 (2005). In Korea, some articles have introduced the history and practice of clinics in U.S. law schools to the Korean academe. They, however, seem to lack details. Jae-Jeong Jun discusses external clinics, whose meaning is not clear. See Jae-Jeong Jun, Theories and Methods of Clinical Legal EducationChallenges and Promise of Korean Clinical Legal Education, 49 SEOUL LAW REVIEW 409, 433 (2008).

${ }^{76}$ The Court Administration Office touted a moot court competition under the auspices of the Korean Supreme Court, which seems odd as civil cases are still dominated by briefs, not oral arguments. See Jeonkuk beophak jeonmun daehakweon beopjang byeonron gyeongyeon daehoireul uihan solmyeonghoi gaech'oi [National law school holds moot court competition before high court], YONSEI LAW SCHOOL, available at http://lawschool.yonsei.ac.kr/contents/board/notice.html?pkid=57.
} 
forty-six out of ninety credits would be mandatory, while forty-four optional. In general, the mandatory courses of all law schools listed in Table 4 are well structured to make students acquire the basic legal knowledge of the system. On the other hand, considering the number of students, many plans offer rather excessively multiple elective courses.

\section{Global Courses}

The word "global" has implications on the composition of the faculty, the student body, the content of courses, the delivery mode of courses and applicable disciplines. The current discussion in Korean law schools seems to be limited to the delivery mode of courses, that is, courses in English. As certain faculty members have studied in English-speaking countries, they can lead courses in English. At YLS, twenty-six courses will be offered in English. Students, however, are all native Koreans with only twenty-two foreign college graduates out of two thousand for the class of 2012. ${ }^{77}$ International law is one of eight clusters composed of six basic and sixteen advanced courses. At YLS, three credits in one international course are required to graduate.

Nonetheless, more diverse international courses should be offered such as Islamic, Jewish, or Russian law, since the current curriculum on foreign law is largely limited to the U.S., the E.U. and China. Furthermore, more renowned foreign professors should be invited to teach these foreign law and international law study courses. Although it is true that several foreigners are teaching foreign or international law courses at Korean law schools, the excitement they cause originates from their nationality, not their academic achievement. Without renowned foreign faculty, at least for the time being, no Korean law school will become a global education institution, or even a well-regarded one. If unaffordable financial or cultural resources are required to invite foreign faculty members, Korean law schools

\footnotetext{
${ }^{77}$ See Soon-Hyuk Lee, supra note 40.
} 
should seriously consider instituting study abroad programs under which law school students can go to foreign law schools for one or two semesters to study specific subject matter. ${ }^{78}$ In addition to foreign faculty members, the student body should be more diversified in the near future. ${ }^{79}$ This issue, however, should be linked to the LEET and bar examinations and languages as a mid-term goal.

\section{Litigation Versus Non-Litigation}

The difference between dispute resolution and dispute prevention classes is not always clear-cut. Among practice courses, however, legal writing courses are centered around legal briefs and other litigation documents. ${ }^{80}$ Among twelve applied practice courses, seven courses pertain to dispute resolutions, while five pertain to contract drafting and advisory practice. Including one negotiation course as one of basic law cluster, the courses on dispute prevention are six in total, which is almost the same number as dispute resolution courses.

Basic legal skills, such as analysis and communication, would have to be added as part of legal writing courses or as an independent course. Otherwise, non-litigation lawyers would have no chance to acquire legal skills in drafting and advising in a non-litigation context. Applied practice courses, such as intellectual property, labor and mergers \& acquisitions, also should be emphasized. Although such courses are based on specific cases that were disputed in courts, the focus of such substantive law courses should be finding the right law and thus preventing disputes beforehand. In this sense, contract drafting and negotiations should be another pivotal building block of the standard law school curriculum.

\footnotetext{
${ }^{78}$ At this stage of law school development in Korea, as a matter of fact, having full time faculty members for foreign laws might be burdensome to law school administration.

${ }^{79}$ LEET should be open to foreign students who are not proficient at Korean language, who can then take the bar exam in English.

${ }^{80}$ Law School Law Enforcement Decree, Art. 13, Para. 1, Item 3 lists court decisions, complaints and briefs as legal documents to be taught.
} 


\section{StRATEGIES FOR SUCCESSFUl InStitutionALIZATION OF LAW SCHOOLS IN KoreA}

\section{A. What is Success?}

Defining success is the first step in planning the next couple of years at Korean law schools. The Law School Law sets forth the purpose of the legal education at law schools as the production of lawyers who can prevent and resolve complex legal disputes. The lawyer's role, however, is not restricted to disputes. At the beginning of the $20^{\text {th }}$ century, when the Western legal system was first introduced into Korea via Japan, legal education purported to educate judges and prosecutors, who would later enter private practice upon retirement. Even after independence in 1945, the Korean judicial system continued to be boxed into the colonial framework. According to the Law School Law, the goal of the Korean education system should not be centered on legal disputes, but should be focused on the value of good lawyering. The value of lawyering was summarized long before in the MacCrate Report as problem solving, legal analysis and reasoning, legal research, factual investigation, communication, counseling, negotiation, organization and management of legal work, and recognizing and resolving ethical dilemmas in addition to litigation. ${ }^{81}$ The success of the ongoing revolution in legal education in Korea will depend on whether it can produce good lawyers with the skills, knowledge and ethics necessary to achieve the above. ${ }^{82}$

\section{B. Change in Values: The Lawyer as Professional}

A typical lawyer in Korea practices law in three capacities: as a public official; as a business person; and as a member of the intellectual gentility. When new lawyers are

${ }^{81}$ See Am. Bar Assoc., Rep't of the Task Force on L. Schools and the Profession: Narrowing the Gap (Jul. 1992), available at

http://www.abanet.org/legaled/publications/onlinepubs/maccrate.html\#A.\%20Organization\%20of\%20the\%20St atement.

${ }^{82}$ One foreign observer pointed out two potential problems: Confucian values and language. See Jasper Kim, Socrates v. Confucius: An Analysis of South Korea's Implementation of the American Law School Model, 10 ASIAN-PAC. L. \& POL'Y J. 322, 334-50 (2009). 
selected by the fiercely competitive bar examination, the lawyers evaluated as most competent by existing bureaucrats tend to start their career as judges or public prosecutors. Most of them, however, eventually quit their career to start private practice, which is largely a business, not a profession. In the past, most ex-judges and ex-prosecutors were tempted to start as solo practitioners largely for financial reasons, although in recent years many have tended to move towards membership in a firm. As their relations with the officials at courts or the prosecutor's office weakens, they end up with managing offices without any professional services to be rendered. ${ }^{83}$

Since the law school system has been introduced, it has not been clear whether law school graduates can start their career at courts or the public prosecutor's offices, or whether they will only be able to enter private practice. ${ }^{84}$ As in the past, the courts and prosecutors may wish to continue to recruit the best graduates. However, these new graduates most likely have no training in drafting court decisions or letters of indictment. Thus, they will need some vocational training for certain period at courts or prosecutors' offices after passing the bar examination. Assuming that the demand for public officials does not drastically increase in the near future, more graduates will have to start as lawyers trained with legal skills but with less substantive legal knowledge. If so, their value will be not in having the authority to make official decisions at courts or prosecutors' offices, but to analyze the facts, research the relevant laws and suggest the right course of actions as a legal professional. Their income or prestige might be substantially less than that of current licensed lawyers. They, however, will

\footnotetext{
${ }^{83}$ In Japan, there is no such phenomenon because of jurists' commitment to lifetime tenure. Nonetheless, at least one Japanese scholar sees the rule of law as a value advanced by judicial reform. See Setsuo Miyazawa, The Politics of Judicial Reform in Japan: The Rule of Law at Last?, 1 ASIAN-PAC. L. \& POL'Y J. 88 (2001).

84 The Judicial Reform Commission, which will be convened beginning in early February 2010, will finalize the plan soon. See Sabeopbu insa shiseut'em hawk tteuteo goch'inda [Commission to confirm judicial reform], YEONHAP NYUSEU, Jan. 26, 2010, available at http://app.yonhapnews.co.kr/yna/basic/article/Search/YIBW_showSearchArticle.aspx?searchpart=article\&searc htext $=\%$ ec\%82\%ac\%eb\%b2\%95\%ec\%a0\%95\%ec\%b1\%85\%ec\%9e\%90\%eb\%ac\%b8\%ec\% $\%$ c $\% 84 \%$ ec $\% 9 b \% 9$ $0 \%$ ed $\% 9 \mathrm{a} \% 8 \mathrm{c} \&$ contents_id=AKR20100126041400004.
} 
be respected as professionals. As such, they should try to find their purpose in the profession. $^{85}$

\section{Systemization of the Bar Examination}

If the bar examination is taken solely to assess legal education at law schools, the curriculum for higher legal education for professional lawyers would be infeasible. If the bar examination covers many different subjects, the students might fret over the uncertainty of the bar examinations after graduation. Law school faculty members who used to exert their efforts to increase the bar passage rate might similarly be neurotic about the bar exam while teaching their courses at law schools. To emancipate law schools and their students from the fear of bar examination, the examination should be systemized and standardized.

Systemization means that if students study for only one to two months after graduation, they should be able to pass the examination. Standardization means the tests are not skewed or imbalanced, but rather that they are common basic tests can be taken without undue hardship. This whole process will take some time. However, it is the key to the success of law school education. ${ }^{86}$ Fortunately, the Justice Department tried a mock bar exam from January 18-22, 2010 with one hundred and sixty law school students, passers of the fifty-first annual bar exams and the students at the JRTI. ${ }^{87}$ The Justice Department also opened a website for the bar exams to be administered in the spring of 2012. This is a good sign that the Justice Department is taking this issue seriously and is open to public comment.

\footnotetext{
${ }^{85}$ For the situation in Japan, see Setsuo Miyazawa, Education and Training of Lawyers in Japan $-A$ Critical Analysis, 43 S. TEX. L. REV. 491, 492 ("Japanese lawyers have not established themselves as a profession....").

${ }^{86}$ For the details of the Enforcement Decree under the Bar Examination, see Public Notice No. 200983, Korean Ministry of Justice (May 27, 2009).

${ }^{87}$ See Press Release, Byeonhosa shiheom moeui shiheom shilshi [Bar exam simulation test completed], Jan. 18, 2010, available at http://www.moj.go.kr/HP/COM/bbs_03/ListShowData.do?strNbodCd=noti0005\&strWrtNo=2039\&strAnsNo= A\&strNbodCd=noti0005\&strFilePath $=$ moj/\&strRtnURL=MOJ_30200000\&strOrgGbnCd=100000\&strThisPage $=1 \& \operatorname{str}$ NbodCdGbn $=$.
} 


\section{Programming of Legal Education}

Many law schools have devised diverse courses in a haste to earn high scores in the application phase. They are now in the implementation phase. In this phase, they will have to provide guidance to students regarding their academic experience for the remaining two years of law school and their professional lives thereafter. For students to consider their academic experiences and professional lives rewarding, it is not enough to offer several hundreds of diverse theory courses to students. The courses should be streamlined for the various roles of lawyers within the society as well as for each applicable subject. Thus, based on informed decisions, each student can concentrate his or her study on a specific discipline for a chosen career path in the near future. For effective guidance to be given to students, the curriculum among law schools should be focus on offering more practical courses, while theory courses should be reorganized and more non-litigation courses should be developed. ${ }^{88}$

\section{E. Addressing Concerns About the Quality of Lawyers: Continuing Legal Education as Part of Law School Programs}

The Korean Lawyers Law was revised effective July 27, 2007 to prescribe, among other things, eight mandatory hours of continuing legal education per year for bar members. ${ }^{89}$ Continuing legal education can be completed in various ways, such as by attending sessions at the Lawyers Training Center at the Korean Bar Association, ${ }^{90}$ attending conferences of various academic societies, or participating in education programs at academic institutions. Because the concept of continuing legal education is still new to the legal profession in

\footnotetext{
${ }^{88}$ One big question is how to appoint judges and public prosecutors after the JRTI closes its door in 2020. It appears Korea will take the similar course to Japan. For an in-depth look at that path, see Yukio Yanagida, A New Paradigm for Japanese Legal Training and Education - In Light of the Legal Education at Harvard Law School, 1 ASIAN-PAC. L. \& POL'Y J. 1, 31 (2000). Only those who have been practicing attorneys for a specified time, e.g., five years, would be eligible.

${ }^{89}$ See Byeonhosa beop i gaejang [Amendment to the Lawyers Law], Law No. 8271 of 2007, Art. 85, Para. 1. Art. 17-2 of the Lawyers Law Enforcement Decree provides one hour on legal ethics and seven hours of practice.

${ }^{90}$ The Korean Bar Association has been aggressively running almost monthly training programs. For details, see Korean Bar Assoc., Yeonsu annae [Guide to Trainings], http://www.koreanbar.or.kr/lawyer/training_info.asp.
} 
Korea, it remains to be seen how things will unfold. Among these institutions, it is not certain who will play a leading role in the future. One possible and logical option is to have law schools supply new theoretical and practical perspectives to young practicing lawyers. Considering the prospective competitive pressure among new lawyers and the high priority maintaining relevance to the legal profession, Korean law schools will have to and should be able to provide lawyers with institutional support so that they can meet the challenges of changing realities in the legal world. ${ }^{91}$ Several renowned law schools are running short-term programs for a diploma or a master's degree for licensed or non-licensed lawyers, largely for financial reasons. ${ }^{92}$ These programs should be constructively developed into a new education program only for newly licensed lawyers. Otherwise, it would be a waste of law schools' resources.

\section{F. $\quad$ Adaptation of General Graduate Study Programs within Law Schools}

While law colleges with new law school charters are prohibited from recruiting new undergraduate law students, they are allowed to maintain their current graduate law schools. Graduate law schools in Korea largely consist of students who continue to attempt to pass the national bar examinations or wish to pursue a career in academia. As the law school system is instituted, the graduate study program will tend to serve as a bridge for law college graduates who are preparing to enter law schools. High-level legal scholarship should be continued, by all means, even with the implementation of the law school system. At the same time, graduate programs should not devolve into preparatory courses for law schools. Thus,

\footnotetext{
${ }^{91}$ This seems to be the case in the case of Singapore, where robust CLE programs are available. See, e.g., NUS: Faculty of Law - Continuing Legal Education, http://aw.nus.edu.sg/cle/.

${ }^{92} \mathrm{SNU}$, one of the most prestigious schools in Korea, runs one semester programs for a specific subject matters, such as antitrust law, investment banking, corporate finance and M\&A, among others. These programs, however, are open to the general public. As a matter of fact, most of the participants are non-lawyers. See, e.g., Seoul Nat'1 Univ., "M\&A eui irongwa shilmu" gwajeongsu gangsaeng mojip annae ["M\&A theory and practice" course application information for students], http://law.snu.ac.kr/intro/intro_board_view.asp?idx=445\&no=265\&pn=1\&sn=5.
} 
in the near future, graduate programs at law schools should be developed into highly selective judicial doctorial programs for top law school graduates. ${ }^{93}$

\section{G. Alliance with Foreign Law Schools}

While "all politics is local," ${ }^{94}$ all legal issues are global. As the world economic and political community is globalized, regardless of whether such globalization arose from the pressures of speculative capitalism, the resulting benefits to global community or the changing values of humankind, legal issues in one country will be linked to those in other countries. As such, without an understanding of such issues in the international context, no full picture of the issues or the solutions thereto will be available. Law students should be given a chance to study abroad to obtain direct and expert knowledge in local issues. If they want to practice law in another jurisdiction, they should be given the opportunity to sit for the local bar exam. To do so, Korean law schools should exert their efforts to forge alliances with foreign law schools which are willing and able to offer one-semester or one-year terms of study to Korean law students. ${ }^{95}$ The significance of such study might vary depending on the jurisdiction. Students should be able to obtain full credit for degrees from Korean law schools or joint degrees with foreign educational institutions. In addition to gathering experience in a foreign jurisdiction, they could utilize such opportunities to pass local bar

\footnotetext{
${ }^{93}$ SNU seems to be the only law school that has proposed one year SJD program as an extension of law school education. It is also not clear what other law schools would do with their graduate study programs.

94 Thomas P. O’Neill with Gary Hymel, All Politics is Local: AND Other Rules Of THe GAME (1993).

${ }^{95}$ Many law schools have signed MOUs with foreign law schools; however, their meaning is not clear. For example, one can see a list of foreign universities with which YLS has been cooperating. The only significant program that the college has operated is an array of joint LL.M. programs with American University and Washington University for several graduate students at the General Graduate Program. How these programs will evolve for graduate students is not clear. One possibility is to offer the current joint degree program to YLS students. If so, they can secure one LL.M. degree from YLS and another LL.M. degree from AU or WU. However, it is uncertain why Korean law school students would wish to get an LL.M. degree from a U.S. law school after they spend one more year in the United States directly after law school. The only nominal incentive could be to get a bar membership from the State of New York or California. However, unless they wish to start their practice there, the bar membership itself would not have any practical value.
} 
examinations in other countries. Such an extension of the borders of legal education could start from Korean law schools. ${ }^{96}$

\section{CONCLUSION}

New systems cannot always avoid the ill wishes and criticism of naysayers who are afraid of change. The most serious threat to the success of the new system might therefore be current lawyers. Nonetheless, the nation as a whole has decided to swiftly adopt a revolutionary change. In light of Korean history, this is a rare occasion in that the country decided to change without any unavoidable pressure from outside of Korea. ${ }^{97}$ Every success requires some political savvy, however. Among other things, law schools should do their best to recruit top students back to their schools. Otherwise, they may become institutions disregarded by top lawyers, as colleges of law have been in the past. ${ }^{98}$

Furthermore, law schools should make concerted efforts to reorganize the curriculum in line with the goals of law schools. The minimal efforts which are made through the Association include LEET examinations, standard textbook development for certain courses, and seminars with U.S. instructors on clinical or legal research and writing courses. ${ }^{99}$ More attention, however, should be paid to the development and structure of the curriculum, not necessarily via the Association, but via the competitive tension among law schools for the best students. The competition should not be for the size of the faculty, the pure number of

\footnotetext{
${ }^{96}$ Some commentators have brought up this possibility for Japanese law schools, as well. See Luke Nottage, et al., Beyond Borders in the Classroom - The Possibility of Transnational Legal Education, 25 RITSUMEIKAN L. REV. 183 (2008).

${ }^{97}$ Korean law schools may now be able to procure financial assistance from outside sources. See James E. Moliterno, Exporting American Legal Education, 58 J. LegAL EduC. 274 (2008). The ABA has supported the establishment of legal educational systems in Georgia, Armenia and Thailand. Moliterno's work in Japan was funded by the Japanese government. Furthermore, the U.S. State Department has been distributing generous grants to many U.S. law schools for their education programs for emerging capitalist countries, such as Indiana University Law School's Adilet Law School project in Almaty, Kazakhstan. See Ctr. for Internat'l Educ. and Dev. Assistance, Adilet Law School [sic] Almaty, Kazakhstan, http://www.indiana.edu/ ird/cieda/adilet.htm (last visited Apr. 7, 2010).

${ }^{98}$ Sang-Hyun Song, supra note 1, at 399 ("[T] hey are. . . considered second-class citizens within the legal profession because they generally do not take or pass the bar exam.").

${ }^{99}$ More information is available at Beophak jeonmun daegakwon hyeop euihui [Korean Association of Law Schools], http://info.leet.or.kr/ (last visited Apr. 7, 2010).
} 
courses, or the number of students who pass the bar exam, but for the quality of educational services for good lawyers. At this juncture, however, it should be pointed out that law schools are not only for legal education but also for legal scholarship. ${ }^{100}$ Law professors must meet this challenge for the next several years. Only with top notch scholars on their faculties can the new law schools be reborn and remain as the rightful legal institutions in Korea. It is rather unfortunate that Korean law schools must demonstrate the good of the system before it will be permitted to increase the number of students. ${ }^{101}$ After the first phase change is completed in 2012, however, law schools should be able to secure the firmer support of the public when arguing for an expansion of their class sizes.

${ }^{100}$ Yoshida, supra note 16, at 220 ("Law professors struggle to balance a commitment to scholarship with a commitment to training, and many law school professors have overloaded schedules."). The situation in Korea looks similar. Many law professors in Korea seem to wear a number of hats: secretary, lecturer, conference organizer, administrator, researcher, writer, journal editor, office sweeper, tea server, mailman and more.

${ }^{101}$ The situation in Japan is not different. See Yoshiharu Kawabata, The Reform of Legal Education and Training in Japan: Problems and Prospects, 43 S. TEX. L. REV. 419, 434 (2002) ("The most contentious issue [is] how many people should be permitted to sit for the National Bar."). As undergraduate level legal education continues simultaneously with graduate schools, Japan has much more complicated goals for each institution. Although it is too early to tell about the future of Japanese law schools, many seem to have closed soon after the opening. For a U.S. practitioner's outcry for more lawyers in Japan, see Robert F. Grondine, An International Perspective on Japan's New Legal Education System, 2 ASIAN-PAC. L. \& POL'Y J. 13 (2001). 